\title{
HOMOGENIZATION OF THE CRITICALITY SPECTRAL EQUATION IN NEUTRON TRANSPORT
}

\author{
Grégoire Allaire $^{1}$ And Guillaume BaL ${ }^{2}$
}

\begin{abstract}
We address the homogenization of an eigenvalue problem for the neutron transport equation in a periodic heterogeneous domain, modeling the criticality study of nuclear reactor cores. We prove that the neutron flux, corresponding to the first and unique positive eigenvector, can be factorized in the product of two terms, up to a remainder which goes strongly to zero with the period. One term is the first eigenvector of the transport equation in the periodicity cell. The other term is the first eigenvector of a diffusion equation in the homogenized domain. Furthermore, the corresponding eigenvalue gives a second order corrector for the eigenvalue of the heterogeneous transport problem. This result justifies and improves the engineering procedure used in practice for nuclear reactor cores computations.

Résumé. On considère l'homogénéisation d'un problème aux valeurs propres pour l'équation du transport neutronique dans un milieu hétérogène périodique qui modélise l'étude de la criticité d'un cœur de réacteur nucléaire. On démontre que le flux neutronique, correspondant à l'unique premier vecteur propre, peut se factoriser en un produit de deux termes, à un reste près qui tend vers zéro avec la période. Un des termes est le premier vecteur propre de l'équation du transport dans la cellule de périodicité. L'autre terme est le premier vecteur propre d'une équation de diffusion dans un milieu homogénéisé. De plus, la valeur propre correspondante est un correcteur du deuxième ordre pour la valeur propre du problème de transport hétérogène. Ce résultat justifie et améliore les formules utilisées en pratique par les ingénieurs pour le calcul des cœurs de réacteurs.
\end{abstract}

AMS Subject Classification. 35B27.

Received: February 17, 1998. Revised: July 8, 1998.

\section{INTRODUCTION}

The power distribution in a nuclear reactor core is usually determined by solving a transport equation for the neutron flux. In many practical situations in reactor analysis, only a steady-state solution is required, and the time variable is eliminated. The steady equation is of a non-standard type since the source term is itself a function of the solution (neutrons are produced by fission which itself is triggered by other neutrons in a chain reaction). This time-independent transport equation is a linear eigenvalue problem which is called the criticality problem for the neutron transport equation. It expresses the balance between the production

Keywords and phrases. Homogenization, transport equations.

1 Laboratoire d'Analyse Numérique, Université Paris VI, 75252 Paris Cedex 5, France, and CEA Saclay, DRN/DMT/SERMA, 91191 Gif-sur-Yvette, France. e-mail: allaire@ann.jussieu.fr

2 Électricité de France DER/IMA/MMN, 92141 Clamart, France and LAN, Université Paris VI, 75252 Paris Cedex 5, France. Current address: Stanford University, Department of Mathematics, CA 94305, USA. e-mail: bal@math.Stanford.edu 
of neutrons by fission and its absorption or scattering in the reactor core and leakage at the boundary. The unknowns are not only the neutron flux $\phi(x, v)$, which is the neutron density at point $x$ with velocity $v$, but also the positive ratio $k_{\text {eff }}$ (the so-called multiplication factor) which measures the balance between the production and removal of neutrons. More precisely, $\left(\phi(x, v), k_{\text {eff }}\right)$ is a couple of eigenvector and eigenvalue for a transport equation. Since only positive densities have a physical meaning, the only relevant solution turns out to be the first positive eigenvector (positive and unique up to a multiplicative constant). Therefore, $\phi(x, v)$ is the first eigenvector and $1 / k_{\text {eff }}$ is the smallest eigenvalue satisfying the following equation

$$
v \cdot \nabla \phi+\Sigma(x, v) \phi=\int_{V} f\left(x, v^{\prime}, v\right) \phi\left(x, v^{\prime}\right) \mathrm{d} v^{\prime}+\frac{1}{k_{\mathrm{eff}}} \int_{V} \sigma\left(x, v^{\prime}, v\right) \phi\left(x, v^{\prime}\right) \mathrm{d} v^{\prime}
$$

with appropriate boundary conditions in a convex bounded open set $\Omega \subset \mathbb{R}^{N}$ and with a compact velocity space $V \subset \mathbb{R}^{N}$. The coefficients in (1) are called cross sections: $\Sigma$ is the total (or absorbing) cross section, $f$ is the scattering cross section, and $\sigma$ is the fission cross section.

The interpretation of the multiplication factor $k_{\text {eff }}$ is as follows (for details, see e.g. $[12,15,27]$ ): if it is equal to 1 , there is a perfect balance between production and removal of neutrons and the reactor is said to be critical; if it is larger than 1, then too many neutrons are produced and the reactor is super-critical (it can operate only if absorbing control rods are introduced in the core); if it is smaller than 1, then not enough neutrons are produced and the reactor is sub-critical (the fission chain reaction dies out). On the other hand the solution $\phi(x, v)$ of (1) indicates the relative power distribution in the core. Remark that, since it is an eigenvector, $\phi(x, v)$ is defined up to a multiplicative constant, and therefore the total power in the core is not given by the criticality problem (1).

Solving numerically the eigenvalue problem (1) in a whole nuclear core is still a challenge with modern computers, even in two space dimensions. One striking reason is that nuclear cores, defined through the cross section $\Sigma, f$ and $\sigma$, are highly heterogeneous, asking for a very fine mesh. However, the periodic structure of many usual cores allows for first homogenizing the transport equation, and then solving numerically the homogenized problem. In practice the homogenized problem is a diffusion equation with slowly varying coefficient, which is much easier to solve numerically. This homogenization problem has been widely addressed in the past forty years in physics (see e.g. $[10,12,15]$ ) as well as in mathematics (see e.g. $[11,20,21,27,29]$ ). In the physical and mathematical literature the methodology is always the same: the neutron flux $\phi(x, v)$, solution of (1), is factorized as the product of two terms

$$
\phi(x, v)=\psi(x, v) u(x),
$$

where $\psi(x, v)$ is the solution of the so-called infinite medium transport equation, and $u(x)$ is the solution of an homogenized diffusion equation. The infinite medium transport equation is the same criticality spectral equation than (1), but posed in a single periodicity cell with periodic boundary conditions. On the other hand, the homogenized diffusion equation is also a spectral problem posed in the whole core (much simpler than (1) since it does not involve a velocity variable). The macroscopic diffusion flux $u(x)$ gives the rough shape of the true flux $\phi(x, v)$, while the microscopic transport flux $\psi(x, v)$ corresponds to the local oscillations of the true flux. This factorization procedure is at the basis of most numerical computations of reactor cores. Of course, a crucial problem for engineers is to know how to compute the homogenized coefficients for the diffusion equation (there are many available formulas, see for example the so-called Benoist formula in Rem. 3.3).

In this paper we rigorously justify this factorization principle for a periodic domain. We also furnish formulas for the homogenized diffusion coefficients and an asymptotic expansion for the criticality eigenvalue $1 / k_{\text {eff }}$ (see Th. 3.1). Our homogenization formulas differ from those in the physical literature, but coincide with those found by Larsen [20,21]. There are two main differences between the works of Larsen and ours. First, Larsen studied a time-dependent transport equation instead of the spectral problem (1) with an assumption of local criticality on the cross-sections. Second, following his previous work with Keller [22], he uses formal two-scale asymptotic expansions to derive a homogenized limit, without rigorous convergence theorem. Of course, the geometrical 
assumption of periodicity is hardly satisfied by modern reactors. However, it is up to now essentially the only case where homogenization can be rigorously justified either physically or mathematically. The treatment of heterogeneous cores with different types of assemblies (i.e. with a slowly varying periodicity cell) is the subject of active current research. The factorization principle is not so clear in this case (see Rem. 3.5) and boundary layers may appear between different cells. However, under further assumptions, we expect that a suitable generalization of our approach will succeed also for a piecewise periodic core.

We now discuss our scaling assumptions for homogenizing the spectral transport equation (1). As usual, the unit periodicity cell is denoted by $Y=(0,1)^{N}$. The reactor core is a bounded domain $\Omega$. Assuming that $\Omega$ is periodic, we denote by $\varepsilon>0$ the periodicity of the heterogeneities in $\Omega$. Since the size of the domain is independent of $\varepsilon, \Omega$ is composed of the order of $\varepsilon^{-N}$ periodicity cells of the type $\varepsilon Y=(0, \varepsilon)^{N}$. Homogenization is an asymptotic analysis as the small parameter $\varepsilon$ goes to 0 , or equivalently as the number of cells grows up to infinity. Since $\varepsilon$ is the size of the periodicity cell and the physical unit of cross sections is the inverse of a length, one must carefully scale the cross sections in terms of $\varepsilon$. The mean free path of the neutrons is physically independent of the number of unit cells in the core; therefore it must remain of the same size as the unit cell. Accordingly, in order to perform a consistent asymptotic analysis we have to scale all cross sections to be of the order of $1 / \varepsilon$. Therefore, introducing $\lambda_{\varepsilon}=1 / k_{\text {eff }}$, equation (1) is replaced by the following sequence of criticality problems

$$
\left\{\begin{array}{l}
\varepsilon v \cdot \nabla \phi_{\varepsilon}+\Sigma^{\varepsilon}(x, v) \phi_{\varepsilon}=\int_{V} f^{\varepsilon}\left(x, v^{\prime}, v\right) \phi_{\varepsilon}\left(x, v^{\prime}\right) \mathrm{d} v^{\prime}+\lambda_{\varepsilon} \int_{V} \sigma^{\varepsilon}\left(x, v^{\prime}, v\right) \phi_{\varepsilon}\left(x, v^{\prime}\right) \mathrm{d} v^{\prime} \quad \text { in } \Omega \times V \\
\phi_{\varepsilon}=0 \quad \text { on } \Gamma_{-}=\{(x, v) \in \partial \Omega \times V \mid v \cdot n(x)<0\}
\end{array}\right.
$$

where the cross sections are periodic functions given by

$$
\Sigma^{\varepsilon}(x, v)=\Sigma(x / \varepsilon, v), f^{\varepsilon}\left(x, v^{\prime}, v\right)=f\left(x / \varepsilon, v^{\prime}, v\right), \sigma^{\varepsilon}\left(x, v^{\prime}, v\right)=\sigma\left(x / \varepsilon, v^{\prime}, v\right)
$$

with $\Sigma, f$ and $\sigma$ positive $Y$-periodic functions of the space variable, and independent of $\varepsilon$ (see section 2 for more detailed assumptions). The absorbing boundary condition in (2) expresses that no particles enter the core. This statement is physically valid as a first approximation. It can be shown (at least formally) that the addition of a dissipative reflector around the core merely modifies the neutron density by an amount of the order of $\varepsilon$.

The paper is organized as follows. In the next section basic mathematical properties of problem (2) are recalled, concerning the existence and regularity of its solutions when $\varepsilon$ is fixed. It also encompasses the criticality eigenvalue problem with periodic boundary conditions. Section 3 is devoted to a detailed presentation of our main results concerning the homogenization of (2). It includes the factorization of the neutron flux and a second order asymptotic expansion for the associated eigenvalue. The proofs of these results are given in Sections 4 and 5. More precisely, Section 4 focuses on a priori estimates for a source problem associated with (2), while Section 5 is concerned with the proof of the homogenization process, using the two-scale convergence method. Finally, Section 6 contains some corrector results and numerical computations for assessing our homogenization theorem. Our results have been announced in [3]. Similar homogenization theorems have been proved for the criticality spectral problem when using a diffusion model instead of our transport equation [4,5,24]. In different contexts, various homogenization results have been obtained for transport equations (see e.g. $[1,16,17,19])$.

\section{Existence AND REGUlARITy RESUlts FOR EIGENVALUE PROBLEMS IN TRANSPORT}

The goal of this section is to establish some results concerning the existence and the regularity of eigenvalues and eigenvectors for our model of neutron transport. Although the following theorems are mostly variations of previously known results, to the best of our knowledge they have not appeared elsewhere. Since they are the starting point of our analysis in the next sections, we include them in this paper for the sake of completeness. Most of the proofs below are merely sketched and we refer to the thesis of the second author [7] for complete details. The reader who is willing to accept such results can safely skip this section in a first pass. 
We first give the detailed assumptions on the phase space and the coefficients of the transport equation that are used throughout this paper.

(H1) The domain $\Omega$ is a convex bounded open set.

(H2) The velocity space $V$ is a compact subset of $\mathbb{R}^{N}$ which does not contain 0 . Furthermore $V$ is assumed to be the closure of an open set, and its $N$-dimensional measure is normalized to have $|V|=1$.

(H3) The cross-sections $\Sigma(y, v), f\left(y, v^{\prime}, v\right), \sigma\left(y, v^{\prime}, v\right)$ are measurable positive bounded functions of their arguments and there exists a positive constant $C>0$ such that, for a.e. $\left(y, v, v^{\prime}\right)$,

$$
\begin{aligned}
\sigma\left(y, v^{\prime}, v\right) & \geq C \\
\Sigma(y, v)-\int_{V} f\left(y, v^{\prime}, v\right) \mathrm{d} v^{\prime} & \geq C \\
\Sigma(y, v)-\int_{V} f\left(y, v, v^{\prime}\right) \mathrm{d} v^{\prime} & \geq C .
\end{aligned}
$$

(H4) Denoting by $Y=(0,1)^{N}$ the unit cube, the cross-sections $\Sigma(y, v), f\left(y, v^{\prime}, v\right), \sigma\left(y, v^{\prime}, v\right)$ are $Y$-periodic functions with respect to the space variable $y$.

Assumption (H1) is somehow classical: the convexity of $\Omega$ ensures that the boundary condition is physically relevant, i.e. that the trajectory of an outgoing neutron does not enter again in $\Omega$. The first part of (H2) implies that only finite non-zero velocities are considered. Physically, it means that all neutrons either interact with the media (by absorption or scattering) or leave the domain in a finite non-zero time. The second part of (H2), namely that it is the closure of its interior, is a mathematical convenience which allows to define distributions and Sobolev spaces on $V$ (see Chap. 21 in [14]). Actually all results presented here can easily be extended to the multi-group case, i.e. when $V$ is the union of a finite number of spheres centered at the origin. Remark that in both cases $V$ is not reduced to a subset of a hyper-plane of $\mathbb{R}^{N}$, which ensures that a diffusion approximation of transport makes sense.

The first part of assumption (H3) gives the natural functional framework for the coefficients (i.e. they belong to $L^{\infty}(Y \times V)$ ). Indeed, nuclear reactor cores are heterogeneous domains with merely bounded discontinuous cross sections. The second part of (H3) implies that fission occurs everywhere in the phase space and that there is always a net absorption (i.e. the difference between total absorption and scattering is positive).

Finally the periodicity assumption (H4) is crucial for the homogenization procedure. In particular our results do not hold true any longer if the cross-sections are the product of periodic functions with macroscopic modulations, for example $\Sigma(x, x / \varepsilon, v)$ with a $Y$-periodic function $\Sigma(x, y, v)$. Let us mention however that small perturbations of order $\varepsilon^{2}$ of the cross sections can be allowed (for example, $\Sigma(x / \varepsilon, v)+\varepsilon^{2} \Sigma^{\prime}(x, x / \varepsilon, v)$ ). This yields a non essential generalization of the results presented here (see [7]).

Remark 2.1. Assumption (H3) is not completely satisfactory since it implies that fission occurs everywhere, which is certainly not the case in the moderator around the fuel rods. Fortunately, one can replace the first inequality of (3) by the following one

$$
\sigma\left(y, v^{\prime}, v\right)+f\left(y, v^{\prime}, v\right) \geq C, \text { for a.e. }\left(y, v, v^{\prime}\right),
$$

with $\sigma \geq 0$ and $\sigma \neq 0$. This implies merely that fission plus scattering is positive everywhere. Up to some easy technicalities, all our results hold true also with this more physical assumption.

Introducing the Hilbert space

$$
W^{2}(\Omega \times V)=\left\{u \in L^{2}(\Omega \times V) \text { s.t. } v \cdot \nabla u \in L^{2}(\Omega \times V)\right\},
$$


a first existence result is the following

Theorem 2.2. The spectral problem (2) has at most a countable number of eigenvalues and of associated eigenvectors which belong to $W^{2}(\Omega \times V)$. Furthermore, there exists a real and positive eigenvalue, of smallest modulus, with multiplicity one, and such that its associated eigenvector be the unique (up to a multiplicative constant) positive eigenvector of (2).

The proof of Theorem 2.2, which is in the spirit of other results in Chapter 21 of [14], can be found in [7]. It uses two main arguments. First, the solutions of (2) are shown to be the eigenvalues and eigenvectors of a positive compact operator in $L^{2}(\Omega \times V)$, which implies that there are at most a countable number of them (possibly complex and finitely many of them). The compactness is a consequence of assumption (H3) on the cross-sections and of the velocity averaging lemma of [18] (or similar compactness result in [25]). Second, the Krein Rutman theory of positive operators asserts that the spectral radius of this compact operator is a simple eigenvalue and that the corresponding eigenvector is positive. As a consequence of Theorem 2.2, only the first eigenvector of (2) has a physical meaning since, being positive, it can indeed model a density of neutrons.

As we shall see in the sequel, the asymptotic behavior of the eigenvectors of (2) is partly governed by the first eigenvector of another eigenvalue problem, similar to (2), the so-called infinite medium criticality eigenvalue problem. It is defined by the same equation as (2) but posed in the whole space $\mathbb{R}^{N}$, which after rescaling is reduced to an equation posed in the unit cell $Y$ with periodicity boundary conditions. Denoting by $\lambda_{\infty}$ and $\psi$ its first eigenvalue and eigenvector, the infinite medium problem is defined in $Y \times V$ by

$$
\left\{\begin{array}{l}
v \cdot \nabla_{y} \psi+\Sigma(y, v) \psi=\int_{V} f\left(y, v^{\prime}, v\right) \psi\left(y, v^{\prime}\right) \mathrm{d} v^{\prime}+\lambda_{\infty} \int_{V} \sigma\left(y, v^{\prime}, v\right) \psi\left(y, v^{\prime}\right) \mathrm{d} v^{\prime} \\
y \rightarrow \psi(y, v) Y \text { - periodic. }
\end{array}\right.
$$

We shall also need an adjoint problem of (5) which has the same first eigenvalue $\lambda_{\infty}$ with a different first eigenvector $\psi^{*}$. Introducing the adjoint cross-sections $f^{*}\left(y, v^{\prime}, v\right)=f\left(y, v, v^{\prime}\right)$ and $\sigma^{*}\left(y, v^{\prime}, v\right)=\sigma\left(y, v, v^{\prime}\right)$ this adjoint problem is defined by

$$
\left\{\begin{array}{l}
-v \cdot \nabla_{y} \psi^{*}+\Sigma(y, v) \psi^{*}=\int_{V} f^{*}\left(y, v^{\prime}, v\right) \psi^{*}\left(y, v^{\prime}\right) \mathrm{d} v^{\prime}+\lambda_{\infty} \int_{V} \sigma^{*}\left(y, v^{\prime}, v\right) \psi^{*}\left(y, v^{\prime}\right) \mathrm{d} v^{\prime} \\
y \rightarrow \psi^{*}(y, v) Y \text { - periodic. }
\end{array}\right.
$$

For problems $(5,6)$, only the first eigenvalue and eigenvector are used in the sequel. As usual they are defined up to a multiplicative constant, but as a corollary of Theorem 2.2 we can choose them positive (therefore they properly model the neutron flux in an infinite periodic medium).

Theorem 2.3. There exists a common eigenvalue $\lambda_{\infty}$ for both problems $(5,6)$ which is real positive, of smallest modulus, with multiplicity one, and such that its associated eigenvectors $\psi$ and $\psi^{*}$ be positive elements of $W^{2}(Y \times V)$.

Since the smallest eigenvalue $\lambda_{\infty}$ is simple, we easily deduce from the classical Fredholm alternative for compact operators the following

Proposition 2.4. Let $\lambda_{\infty}$ and $\psi$ be the first eigenvalue and eigenvector of (5). Let $S(y, v)$ be a source term in $L^{2}(Y \times V)$. There exists a solution $\varphi(y, v) \in W^{2}(Y \times V)$ of

$$
\left\{\begin{array}{l}
v \cdot \nabla_{y} \varphi+\Sigma(y, v) \varphi=\int_{V} f\left(y, v^{\prime}, v\right) \varphi\left(y, v^{\prime}\right) \mathrm{d} v^{\prime}+\lambda_{\infty} \int_{V} \sigma\left(y, v^{\prime}, v\right) \varphi\left(y, v^{\prime}\right) \mathrm{d} v^{\prime}+S(y, v) \\
y \rightarrow \varphi(y, v) Y-\text { periodic }
\end{array}\right.
$$

if and only if $S$ is orthogonal to the first eigenvector $\psi^{*}$ of (6), i.e. S satisfies the compatibility condition

$$
\int_{Y} \int_{V} S(y, v) \psi^{*}(y, v) \mathrm{d} y \mathrm{~d} v=0 .
$$


Furthermore, if it exists, the solution $\varphi$ is unique up to the addition of a multiple of $\psi$.

Finally, in the next section the first eigenvectors $\psi$ and $\psi^{*}$ are required to be bounded from above and below by positive constants. This is the purpose of the next Proposition which is based on averaging lemmas [18] and Sobolev inequalities.

Proposition 2.5. Let $\psi$ and $\psi^{*}$ be the first positive eigenvectors of problems (5) and (6), respectively. There exists two positive constants $C^{\prime} \geq C>0$ such that, for a.e. $(y, v)$,

$$
0<C \leq \psi(y, v) \leq C^{\prime} \quad \text { and } \quad 0<C \leq \psi^{*}(y, v) \leq C^{\prime}
$$

Proof. To simplify the notations, we recast both problems (5) and (6) as follows (this is possible thanks to assumption (H3)). Let $\varphi(x, v)$ be a non-zero positive solution in $W^{2}(Y \times V)$ of

$$
\left\{\begin{array}{l}
v \cdot \nabla \varphi+\Sigma(x, v) \varphi=\int_{V} \sigma_{\infty}\left(x, v^{\prime}, v\right) \varphi\left(x, v^{\prime}\right) \mathrm{d} v^{\prime} \quad \text { in } Y \times V \\
x \rightarrow \varphi(x, v) Y \text { - periodic, }
\end{array}\right.
$$

where $\Sigma$ and $\sigma_{\infty}$ are $L^{\infty}$ functions which satisfy

$$
\Sigma_{1} \geq \Sigma(x, v) \geq \Sigma_{0}>0, \quad \sigma_{1} \geq \sigma_{\infty}\left(x, v^{\prime}, v\right) \geq \sigma_{0}>0 \quad \text { a.e. in } Y \times V \times V \text {. }
$$

Let us define two functions $g$ and $\tilde{\varphi}$ by

$$
g(x, v)=\int_{V} \sigma_{\infty}\left(x, v^{\prime}, v\right) \varphi\left(x, v^{\prime}\right) \mathrm{d} v^{\prime}, \quad \tilde{\varphi}(x)=\int_{V} \varphi(x, v) \mathrm{d} v .
$$

From these definitions, we easily deduce that

$$
\sigma_{0} \tilde{\varphi}(x) \leq g(x, v) \leq \sigma_{1} \tilde{\varphi}(x)
$$

Furthermore, integrating equation (7) along its characteristics leads to

$$
\int_{0}^{\infty} g(x-s v, v) \mathrm{e}^{-\Sigma_{1} s} \mathrm{~d} s \leq \varphi(x, v) \leq \int_{0}^{\infty} g(x-s v, v) \mathrm{e}^{-\Sigma_{0} s} \mathrm{~d} s .
$$

Here the functions defined on $Y \times V$ have been extended to $\mathbb{R}^{N} \times V$ by periodicity. If we can prove that $\tilde{\varphi}(x)$ and $1 / \tilde{\varphi}(x)$ belong to $L^{\infty}(Y)$, then we deduce from $(8)$ that $g(x, v)$ and $1 / g(x, v)$ belong to $L^{\infty}(Y \times V)$, and from (9) that $\varphi(x, v)$ and $1 / \varphi(x, v)$ belong to $L^{\infty}(Y \times V)$, which is the desired result.

We divide the proof that $\tilde{\varphi}(x)$ is bounded from below and above in two steps: first we show that $\tilde{\varphi}(x)$ belongs to any $L^{p}(Y)$ with $2 \leq p<+\infty$, and second we show that $\tilde{\varphi}(x)$ and $1 / \tilde{\varphi}(x)$ belong to $L^{\infty}(Y)$.

The first step relies on the following averaging lemma [18]. If $\varphi$ and $v \cdot \nabla \varphi$ belong to $L^{p}(Y \times V)$ for $2 \leq p<\infty$, then $\tilde{\varphi}(x)$ belongs to $W^{s, p}(Y)$ for all $0<s<1 / p ; W^{s, p}(Y)$ is the Sobolev space of functions whose fractional $s$-derivative belongs to $L^{p}(Y)$. By the well-known Sobolev embedding theorem, $W^{s, p}(Y) \subset L^{q}(Y)$ with $q=N p /(N-s p)$, and therefore $\tilde{\varphi}(x) \in L^{q}(Y)$. In other words, we have

$$
\varphi \text { and } v \cdot \nabla \varphi \in L^{p}(Y \times V) \Rightarrow \tilde{\varphi} \in L^{q}(Y) \quad \text { with } p \leq q<\frac{N p}{N-1}
$$

As a consequence of inequality (8), $\tilde{\varphi} \in L^{q}(Y)$ implies that $g \in L^{q}(Y \times V)$, and this is a standard result in transport theory that, if the source term $g$ belongs to $L^{q}(Y \times V)$, then the solution $\varphi$ of equation (7) belongs to $W^{q}(Y \times V)=\left\{u \in L^{q}(Y \times V)\right.$ s.t. $\left.v \cdot \nabla u \in L^{q}(Y \times V)\right\}$. By assumption, we know that $\varphi \in W^{2}(Y \times V)$. 
Using the regularity result (10) and a bootstrap argument, we therefore deduce that $\tilde{\varphi}$ belongs to any $L^{p}(Y)$ with $2 \leq p<+\infty$.

The second step uses assumption (H2) which implies that $V$ is included in a corona $\mathcal{C}$ defined by its radii $0<v_{\min }<v_{\max }<+\infty$

$$
V \subset \mathcal{C}=\left\{v \in \mathbb{R}^{N} \text { s.t. } 0<v_{\min } \leq|v| \leq v_{\max }\right\} .
$$

Integrating (9) over $V$ and using (8) yields

$$
\tilde{\varphi}(x) \leq \sigma_{1} \int_{V} \int_{0}^{\infty} \mathrm{e}^{-\Sigma_{0} s} \tilde{\varphi}(x-s v) \mathrm{d} s \mathrm{~d} v .
$$

Since $V \subset \mathcal{C}$ the change of variables $y=x-s v$ from polar to Cartesian coordinates leads to

$$
\tilde{\varphi}(x) \leq C \int_{\mathbb{R}^{N}} \frac{\mathrm{e}^{-\frac{\Sigma_{0}}{v_{m i n}}|y-x|}}{|y-x|^{N-1}} \tilde{\varphi}(y) \mathrm{d} y
$$

where $C$ is a positive constant which depends on $v_{\min }$ and $v_{\max }$ but not on $\varphi$. By Hölder inequality, for any $\eta>0$ there exists a positive constant $C_{\eta}$ such that

$$
\tilde{\varphi}(x) \leq C\left(\int_{|y-x| \leq 1} \frac{\tilde{\varphi}(y) \mathrm{d} y}{|y-x|^{N-1}}+\|\tilde{\varphi}\|_{L^{2}(Y)}\right) \leq C_{\eta}\left(\|\tilde{\varphi}\|_{L^{N+\eta}(Y)}+\|\tilde{\varphi}\|_{L^{2}(Y)}\right),
$$

since $1 /|y-x|^{N-1}$ belongs to $L^{p}(Y)$ for any $1 \leq p<\frac{N}{N-1}$. We already know that $\tilde{\varphi}$ belongs to $L^{N+\eta}(Y)$ for positive $\eta$, therefore we deduce that $\tilde{\varphi}$, and consequently $\varphi$, is bounded. To show that $1 / \tilde{\varphi}$ belongs to $L^{\infty}(Y)$, we use a symmetric argument. By assumption (H2) the interior of $V$ is non-empty. In particular, there exists a velocity $v_{0}$ and a parameter $\delta>0$ such that the following angular sector $\mathcal{S}$ is included in $V$

$$
\mathcal{S}=\left\{v \in \mathbb{R}^{N} \text { s.t. } 1-\delta \leq \frac{|v|}{\left|v_{0}\right|} \leq 1+\delta, \frac{v}{|v|} \cdot \frac{v_{0}}{\left|v_{0}\right|} \geq 1-\delta\right\} \subset V .
$$

Integrating again (9) over $V$ and using (8) gives

$$
\sigma_{0} \int_{V} \int_{0}^{\infty} \mathrm{e}^{-\Sigma_{1} s} \tilde{\varphi}(x-s v) \mathrm{d} s \mathrm{~d} v \leq \tilde{\varphi}(x) .
$$

Since $\mathcal{S} \subset V$ the change of variables $y=x-s v$ from polar to Cartesian coordinates leads to

$$
C \int_{C_{\mathcal{S}}} \frac{\mathrm{e}^{-\frac{\Sigma_{1}}{v_{\max }}|y-x|}}{|y-x|^{N-1}} \tilde{\varphi}(y) \mathrm{d} y \leq \tilde{\varphi}(x),
$$

where $C_{\mathcal{S}} \subset \mathbb{R}^{N}$ is the infinite cone of origin $x$ defined by $\left\{x+s v\right.$ s.t. $\left.s \in \mathbb{R}^{+}, v \in \mathcal{S}\right\}$. Clearly, at a possibly large, but finite, distance of $x$, this cone contains at least one periodicity cell $Y$. Hence there exists a positive constant $C>0$ such that

$$
C \int_{Y} \tilde{\varphi}(y) \mathrm{d} y \leq \tilde{\varphi}(x)
$$

By hypothesis, $\varphi$ is non-zero and positive in $L^{2}(Y \times V)$. Therefore, $\int_{Y} \tilde{\varphi}(y) \mathrm{d} y>0$ which provides us with a lower bound for $\varphi$. 


\section{MAIN RESULTS}

This section is devoted to the statement of our main results on the asymptotic analysis of the spectral problem (2). We proceed in somewhat reverse order. First we state our final result in Theorem 3.1. The proof relies on an equivalent formulation of the spectral problem (2), which is amenable to homogenization and given in Proposition 3.4. It also relies on the homogenization of a source problem established in Theorem 3.6. Although the latter topic is classical and has been addressed several times (see $[11,14,20,21,29]$ ), we perform our analysis in a quite different way, using the theory of two-scale convergence and therefore allowing for less regular physical data.

Let $\psi$ and $\psi^{*}$ be the first positive eigenvectors of (5) and (6) respectively. We define a so-called drift flux by

$$
J=\int_{Y} \int_{V} v \psi(y, v) \psi^{*}(y, v) \mathrm{d} y \mathrm{~d} v,
$$

which is a constant vector in $\mathbb{R}^{N}$. We require an additional hypothesis on the coefficients of (2), namely that the drift flux is zero

$$
J=0 .
$$

This assumption on (11) is a kind of symmetry condition in phase space. It can be interpreted as an energy flux, which must be locally null, and it implies that there is no neutron drift. In most practical cases this assumption is satisfied. For example, when $V=-V$ (in the sense that $v \in V \Rightarrow-v \in V$ ) and the cross sections do not depend on the velocity variable (this is the so-called one-velocity isotropic case), $J$ is clearly zero whatever the spatial variations of the coefficients because

$$
\psi^{*}(y, v)=\psi(y,-v) \quad \text { a.e. } \quad(y, v) \in Y \times V .
$$

There are other examples where equality (12) is satisfied. Of particular interest is the following case. If $V=-V$, the cross-sections are symmetric with respect to $v$, and the cell $Y$ has cubic symmetry, then the drift flux is again zero (this can be checked readily). In most existing reactors, the cross sections do depend on the velocity variable. Nevertheless, the symmetry condition is always satisfied (at least as a first approximation); therefore condition (11) does not shrink the field of practical applications.

However, as it has been shown by Larsen and Williams in the setting of time-dependent 1-D problems with anisotropic scattering [23], there are cases for which $J \neq 0$. In such a case $J$ indicates a drift direction, along which neutrons propagate, and the asymptotic regime of $(2)$ is different (we suspect that the neutrons concentrate at the vicinity of the set of points $x \in \partial \Omega$ satisfying $J \cdot n(x)<0$, where $n(x)$ is the outward unit normal to $\partial \Omega$ at point $x$ ).

Let us introduce the $\varepsilon Y$ - periodic function $\psi_{\varepsilon}$ defined by

$$
\psi_{\varepsilon}(x, v)=\psi(x / \varepsilon, v)
$$

where $\psi(y, v)$ is the positive first eigenvector of (5).

Theorem 3.1. Assume that the drift flux defined by (11) is $J=0$. Let $\lambda_{\varepsilon}^{k}$ be the $k^{\text {th }}$ eigenvalue of (2) and let $\phi_{\varepsilon}^{k}$ be a normalized associated eigenvector. Then

$$
\lim _{\varepsilon \rightarrow 0} \frac{\lambda_{\varepsilon}^{k}-\lambda_{\infty}}{\varepsilon^{2}}=\nu^{k}
$$

where $\lambda_{\infty}$ is the first eigenvalue of (5) and $\nu^{k}$ is the $k^{\text {th }}$ eigenvalue of the homogenized diffusion problem

$$
\begin{cases}-\nabla \cdot D \nabla u^{k}(x)=\nu^{k} \bar{\sigma} u^{k}(x) & \text { in } \Omega \\ u^{k}(x)=0 & \text { on } \partial \Omega .\end{cases}
$$


Furthermore, up to a subsequence,

$$
\frac{\phi_{\varepsilon}^{k}(x, v)}{\psi_{\varepsilon}(x, v)} \rightarrow u^{k}(x) \text { strongly in } L^{2}(\Omega \times V)
$$

where $u^{k}$ is an eigenvector associated with the eigenvalue $\nu^{k}$. The homogenized fission cross section is given by

$$
\bar{\sigma}=\int_{Y} \int_{V} \int_{V} \sigma\left(y, v^{\prime}, v\right) \psi^{*}\left(y, v^{\prime}\right) \psi(y, v) \mathrm{d} y \mathrm{~d} v \mathrm{~d} v^{\prime},
$$

while the homogenized diffusion tensor $D=\left(D_{i j}\right)_{1 \leq i, j \leq N}$ in (13) is defined by

$$
D_{i j}=-\int_{Y} \int_{V} v_{j} \psi(y, v) \psi^{*}(y, v) \theta^{i}(y, v) \mathrm{d} v \mathrm{~d} y
$$

and the functions $\left(\theta^{i}\right)_{1 \leq i \leq N}$ are the solutions of the cell problems

$$
\left\{\begin{array}{l}
v \cdot \nabla \theta^{i}+Q\left(\theta^{i}\right)=-v_{i} \quad \text { in } Y \times V \\
y \rightarrow \theta^{i}(y, v) Y-\text { periodic }
\end{array}\right.
$$

where the local scattering operator $Q$ is defined by

$$
Q(\theta)=\frac{\theta}{\psi} \int_{V} \sigma_{\infty}\left(y, v^{\prime}, v\right) \psi\left(y, v^{\prime}\right) \mathrm{d} v^{\prime}-\frac{1}{\psi} \int_{V} \sigma_{\infty}\left(y, v^{\prime}, v\right) \psi\left(y, v^{\prime}\right) \theta\left(y, v^{\prime}\right) \mathrm{d} v^{\prime}
$$

with the notation $\sigma_{\infty}\left(y, v^{\prime}, v\right)=f\left(y, v^{\prime}, v\right)+\lambda_{\infty} \sigma\left(y, v^{\prime}, v\right)$.

Remark 3.2. The labeling of the eigenvalues of (2) is made by increasing order of their real part (they may be complex although, in the limit as $\epsilon$ goes to 0 , they are all real). As usual, the eigenvalues of the homogenized diffusion problem (13) are real and labeled by increasing order. Remark also that, for fixed $\epsilon$, the spectral theory asserts that (2) may have only a finite number of eigenvalues, but, as $\epsilon$ goes to 0 , this number increases up to infinity.

In Theorem 3.1 the extraction of a subsequence for the convergence of eigenvectors is only due to the possible multiplicity of the limit eigenvalue $\nu^{k}$. However, in the physically meaningful case of the first eigenvalue $\nu^{1}$, which is simple, we obtain that the whole sequence of normalized positive eigenvectors $\phi_{\varepsilon}^{1} / \psi_{\varepsilon}$ converges to the positive first eigenvector $u^{1}$ of the homogenized problem.

Remark 3.3. Existence and uniqueness of the solutions $\left(\theta^{i}\right)_{1 \leq i \leq N}$ of the cell problems (16) is a consequence of the zero drift-flux assumption $J=0$. Indeed, upon defining $\chi^{i}=\psi \theta^{i}$, it is easily seen that $\chi^{i}$ satisfies

$$
\left\{\begin{array}{l}
v \cdot \nabla \chi^{i}+\Sigma \chi^{i}=\int_{V} f \chi^{i} \mathrm{~d} v^{\prime}+\lambda_{\infty} \int_{V} \sigma \chi^{i} \mathrm{~d} v^{\prime}-v_{i} \psi \\
y \rightarrow \chi^{i}(y, v) Y-\text { periodic }
\end{array}\right.
$$

for which Proposition 2.4 applies: $J=0$ is simply its compatibility condition (or Fredholm alternative). With this new notation, the diffusion tensor $D$ is given by the so-called Kubo formula (see [11])

$$
D_{i j}=-\int_{Y} \int_{V} v_{j} \psi^{*}(y, v) \chi^{i}(y, v) \mathrm{d} v \mathrm{~d} y .
$$

Formula (15) has first been derived by Larsen [20] using formal two-scale asymptotic expansions. As already said in the introduction there are several formulae for the diffusion coefficient of a heterogeneous periodic network of cells in the physical literature (see e.g. [15]). Formula (15) is the only one to be correct asymptotically 
but others (like the well-known Benoist formula) are derived by using conservation or equivalence principle. For the sake of comparison, we recall the uncorrected Benoist formula (see $[10,15]$ ) in the limit of small buckling and for symmetric cells (in which case $D$ is diagonal)

$$
D_{i i}=-\int_{Y} \int_{V} v_{i} \psi(y, v) j^{i}(y, v) \mathrm{d} v \mathrm{~d} y
$$

and the functions $\left(j^{i}\right)_{1 \leq i \leq N}$ are the solutions of

$$
\left\{\begin{array}{l}
v \cdot \nabla j^{i}+Q\left(j^{i}\right)=-v_{i} \text { on } \Omega \times V \\
y \rightarrow j^{i}(y, v) Y \text { - periodic, }
\end{array}\right.
$$

while the cross sections are simply averaged with the weight $\psi$

$$
\bar{\sigma}=\int_{Y} \int_{V} \int_{V} \sigma\left(y, v^{\prime}, v\right) \psi(y, v) \mathrm{d} y \mathrm{~d} v \mathrm{~d} v^{\prime} .
$$

Remark that the difference between our formula and the Benoist one is the factor $\psi^{*}$.

In order to prove Theorem 3.1, we first establish that the original spectral problem (2) is equivalent to another problem (that will be amenable to homogenization) through a factorization principle.

Proposition 3.4. Let $\psi(y, v)$ be the positive first eigenvector of (5). Then, the linear application

$$
\phi(x, v) \rightarrow u(x, v)=\frac{\phi(x, v)}{\psi(x / \varepsilon, v)}
$$

is continuous in $L^{2}(\Omega \times V)$ and has a continuous inverse. Through this change of variables, the original spectral problem (2) is equivalent to

$$
\begin{cases}\frac{1}{\varepsilon} v \cdot \nabla u_{\varepsilon}+\frac{1}{\varepsilon^{2}} Q_{\varepsilon}\left(u_{\varepsilon}\right)=\nu_{\varepsilon} F_{\varepsilon}\left(u_{\varepsilon}\right) & \text { on } \Omega \times V \\ u_{\varepsilon}=0 & \text { on } \Gamma_{-},\end{cases}
$$

where we have defined

$$
\begin{gathered}
\nu_{\varepsilon}=\frac{\lambda_{\varepsilon}-\lambda_{\infty}}{\varepsilon^{2}} \\
Q_{\varepsilon}(u)=\frac{u}{\psi_{\varepsilon}}\left(\int_{V} \sigma_{\infty}^{\varepsilon}\left(x, v^{\prime}, v\right) \psi_{\varepsilon}\left(x, v^{\prime}\right) \mathrm{d} v^{\prime}\right)-\frac{1}{\psi_{\varepsilon}}\left(\int_{V} \sigma_{\infty}^{\varepsilon}\left(x, v^{\prime}, v\right) \psi_{\varepsilon}\left(x, v^{\prime}\right) u\left(x, v^{\prime}\right) \mathrm{d} v^{\prime}\right), \\
F_{\varepsilon}(u)=\frac{1}{\psi_{\varepsilon}} \int_{V} \sigma^{\varepsilon}\left(x, v^{\prime}, v\right) \psi_{\varepsilon}\left(x, v^{\prime}\right) u\left(x, v^{\prime}\right) \mathrm{d} v^{\prime},
\end{gathered}
$$

with the notation $\sigma_{\infty}^{\varepsilon}\left(x, v^{\prime} v\right)=f^{\varepsilon}\left(x, v^{\prime}, v\right)+\lambda_{\infty} \sigma^{\varepsilon}\left(x, v^{\prime}, v\right)$.

Proof. By Proposition 2.5, we know that $\psi(y, v)$ is bounded from below and from above by two positive constants. Therefore, if $\phi_{\varepsilon} \in L^{2}(\Omega \times V)$, we can define $u_{\varepsilon}(x, v)=\phi_{\varepsilon} / \psi_{\varepsilon}$ which also belongs to $L^{2}(\Omega \times V)$. Reciprocally, if $u_{\varepsilon} \in L^{2}(\Omega \times V)$, then $\phi_{\varepsilon}(x, v)=u_{\varepsilon} \psi_{\varepsilon}$ belongs to $L^{2}(\Omega \times V)$. Using the infinite medium equation (5) satisfied by $\psi$, the equivalence between (2) and (17) is just a matter of simple algebra that we leave to the reader. 
Remark 3.5. The idea of the factorization in Proposition 3.4 is familiar in nuclear physics. Basically it amounts to remarking that both functions $\psi_{\varepsilon}$ and $\phi_{\varepsilon}$ satisfy equation (2) on $\Omega \times V$. The only difference between these two solution is their boundary condition. Thus it is expected that both functions behave similarly away from the boundary of $\Omega$. Indeed, this holds true in the sense that $u_{\varepsilon}$ is shown to vary smoothly with respect to the variable $x$ away from the boundary, and to be almost independent of the fast variable $x / \varepsilon$ and the velocity variable $v$.

At first sight, problem (17) does not seem to be much simpler than (2), but it turns out that optimal a priori estimates can be obtained with (17) rather than (2). We also emphasize that the equivalence between (2) and (17) is a consequence of the strict periodicity assumption (H4) and does not hold true any longer if the cross sections are not periodic functions.

The scattering operator $Q_{\varepsilon}$ defined by (18) is the same as the local scattering operator $Q$ defined in Theorem 3.1 after the change of variables $y=x / \varepsilon$. Its effect is to relax the angular flux to its velocity average (see the energy estimates in Sect. 4). It is a linear continuous operator in any $L^{p}(\Omega \times V)$, for $1 \leq p \leq \infty$, which satisfies the following properties

$$
\begin{gathered}
\operatorname{Ker} Q_{\varepsilon}=\left\{u \in L^{p}(\Omega \times V) ; u(x, v)=\int_{V} u(x, v) \mathrm{d} v\right\}, \\
Q_{\varepsilon}(\varphi(x) u(x, v))=\varphi(x) Q_{\varepsilon}(u(x, v)) \text { and } Q_{\varepsilon}(u)=Q_{\varepsilon}\left(u-\int_{V} u(x, v) \mathrm{d} v\right) .
\end{gathered}
$$

The spectral analysis of equation (17) is obtained from the spectral properties of an operator $S_{\varepsilon}$ defined as follows.

$$
\begin{aligned}
S_{\varepsilon}: L^{2}(\Omega \times V) & \longrightarrow L^{2}(\Omega \times V) \\
q(x, v) & \longmapsto u_{\varepsilon}(x, v)
\end{aligned}
$$

where $u_{\varepsilon}$ is the solution of the following source problem associated with (17)

$$
\begin{cases}\frac{1}{\varepsilon} v \cdot \nabla u_{\varepsilon}+\frac{1}{\varepsilon^{2}} Q_{\varepsilon}\left(u_{\varepsilon}\right)=F_{\varepsilon}(q) & \text { on } \Omega \times V \\ u_{\varepsilon}=0 & \text { on } \Gamma_{-} .\end{cases}
$$

The well-posedness of $(21)$ in $L^{2}(\Omega \times V)$ can easily be obtained from standard transport theories by using the a priori estimates of Section 4 (which imply that the scattering kernel $Q_{\varepsilon}$ has well-suited properties). Alternatively we can use the reverse change of variables of Proposition 3.4 and prove that $\phi_{\varepsilon}=\psi_{\varepsilon} u_{\varepsilon}$ is the solution of a source problem analogue to $(2)$ where we replace $\lambda_{\varepsilon} \int_{V} \sigma^{\varepsilon}\left(x, v^{\prime}, v\right) \phi_{\varepsilon}\left(x, v^{\prime}\right) \mathrm{d} v^{\prime}$ by $\psi_{\varepsilon} F_{\varepsilon}(q)$. Then, classical results (see e.g. Chap. 21 in [14]) yield the existence and uniqueness of $\phi_{\varepsilon}$, and therefore $u_{\varepsilon}$, in $W^{2}(\Omega \times V)$.

The eigenvalues of the operator $S_{\varepsilon}$ are exactly the inverses of those of problem (17) with the same associated eigenvectors. From the analysis of the convergence of the sequence of operators $S_{\varepsilon}$ we shall obtain the asymptotic behavior of its spectrum. The following result, which is proved in Section 5, gives the homogenized limit operator.

Theorem 3.6. Assume that the drift flux defined by (11) satisfies $J=0$. Then the sequence of solutions $u_{\varepsilon}(x, v)$ of (21) converges strongly in $L^{2}(\Omega \times V)$ to the unique solution $u(x) \in H_{0}^{1}(\Omega)$ of the following diffusion problem

$$
\begin{cases}-\nabla \cdot D \nabla u(x)=F(q)(x) & \text { in } \Omega \\ u(x)=0 & \text { on } \partial \Omega\end{cases}
$$

where $D=\left(D_{i j}\right)_{1 \leq i, j \leq N}$ is the positive definite constant matrix defined by (15), and $F(q) \in L^{2}(\Omega)$ is given by

$$
F(q)(x)=\int_{Y} \int_{V} \int_{V} \sigma\left(y, v^{\prime}, v\right) \psi^{*}\left(y, v^{\prime}\right) \psi(y, v) q(x, v) \mathrm{d} v \mathrm{~d} y \mathrm{~d} v^{\prime} .
$$


When the drift flux is non zero $(J \neq 0)$, the sequence $u_{\varepsilon}(x, v)$ converges strongly to 0 in $L^{2}(\Omega \times V)$.

Remark 3.7. The homogenization of the source problem (21) fits into the framework of previous works in homogenization of transport equations $[11,14,20,21,29]$. Let us emphasize however that Theorem 3.6 is new for two reasons. First, our proof uses the notion of two-scale convergence instead of two-scale asymptotic expansions: this allows to remove all smoothness assumptions on the coefficients that are simply asked to be measurable and bounded (this is the natural setting for heterogeneous media). Second, the special structure of the scattering kernel $Q_{\varepsilon}$ allows us to homogenize (21) without adding extra absorption as is the case usually. Indeed, we establish in Section 4 that the sequence of solutions of (21) is bounded using a coercivity property of $Q_{\varepsilon}$ combined with a Poincaré-type inequality. Of course, Theorem 3.6 is easily generalized if we add an extra absorption term $\alpha u_{\varepsilon}$ in (21), with $\alpha \geq 0$, which yields an additional term $\alpha u$ in (22).

Assuming for the moment that Theorem 3.6 holds true, we are in a position to complete the

Proof of Theorem 3.1. Let us define the homogenized limit operator $S$ by

$$
\begin{aligned}
L^{2}(\Omega \times V) & \rightarrow L^{2}(\Omega \times V) \\
q(x, v) & \mapsto u(x),
\end{aligned}
$$

where $u$ is the solution of (22-23). Clearly $S$ is a compact operator. We deduce from the homogenization Theorem 3.6 that the sequence $S_{\varepsilon}$ converges to $S$ pointwise in the strong topology of $L^{2}(\Omega \times V)$, i.e.

$$
\forall q \in L^{2}(\Omega \times V) \quad S_{\varepsilon} q \rightarrow S q \quad \text { strongly in } L^{2}(\Omega \times V) .
$$

Furthermore, as a consequence of Corollary 4.2, given in Section 4, the sequence $S_{\varepsilon}$ converges compactly to $S$ in the sense that for any bounded sequence $q_{\varepsilon}$ in $L^{2}(\Omega \times V)$, the sequence $S_{\varepsilon} q_{\varepsilon}$ is relatively compact in $L^{2}(\Omega \times V)$. Finally, Theorem 3.8 below allows to conclude the proof of Theorem 3.1. Remark in passing that the convergence of $S_{\varepsilon}$ to $S$ cannot be uniform in general because, if $q_{\varepsilon}$ converges weakly to $q$ in $L^{2}(\Omega \times V)$, there is usually no reason for $F_{\varepsilon}\left(q_{\varepsilon}\right)$ to converge to $F(q)$.

We now recall a classical result in operator theory that can be found, for example, in [6] or in [13] (see Th. 5.5, Prop. 5.6, Th. 5.10, Th. 5.20, and Prop. 5.28 in [13]).

Theorem 3.8. Let $X$ be a Banach space and $\mathcal{L}(X)$ be the set of bounded linear operators in $X$. Let $\left\{T_{n}\right\}_{n \in \mathbb{N}}$ be a sequence of operators in $\mathcal{L}(X)$ converging compactly to $T$ in the sense that:

- for all $x \in X, T_{n} x \rightarrow T x$ as $n \rightarrow \infty$,

- for any bounded sequence $\left\{x_{n}\right\}_{n \in \mathbb{N}}$ with $\left\|x_{n}\right\| \leq 1$, the sequence $\left\{\left(T-T_{n}\right) x_{n}\right\}_{n \in \mathbb{N}}$ is relatively compact in $X$.

Let $\sigma(T)$ and $\sigma\left(T_{n}\right)$ be the spectra of $T$ and $T_{n}$, respectively. Let $\lambda$ be an isolated eigenvalue of $T$ of finite multiplicity and let $\Gamma$ be a closed Jordan curve in the complex plane around $\lambda$ and isolating $\lambda$ such that the domain $\Delta$ enclosed by $\Gamma$ contain no other point of the spectrum $\sigma(T)$ than $\lambda$. Then, $\sigma\left(T_{n}\right) \cap \Delta$ contains a number of eigenvalues equal to the multiplicity of $\lambda$ provided $n$ is large enough.

Moreover, if $\lambda_{n}$ is a sequence of eigenvalues of $T_{n}$ converging to $\lambda$, and $u_{n}$ is a sequence of normalized associated eigenvectors, then, up to a subsequence, $u_{n}$ converges to a limit $u$ in $X$ which is an eigenvector of $T$ associated with $\lambda$.

\section{A PRIORI ESTIMATES}

The first step in the proof of Theorem 3.6 is to derive an a priori energy estimate for the source problem (21). From this we shall derive two main results: first the solution of (21) is bounded in $W^{2}(\Omega \times V)$ independently of $\varepsilon$, and second the rate of convergence of the angular flux to its mean and of the boundary trace of the flux to 0 is characterized. More precisely, the following a priori estimates hold true. 
Lemma 4.1. Let $u_{\varepsilon}$ be the unique solution of (21). There exists a positive constant $C$, which does not depend on $\varepsilon$ and $q$, such that

$$
\left\|u_{\varepsilon}\right\|_{L^{2}(\Omega \times V)}+\left\|v \cdot \nabla u_{\varepsilon}\right\|_{L^{2}(\Omega \times V)}+\frac{1}{\sqrt{\varepsilon}}\left\|u_{\varepsilon}\right\|_{L^{2}\left(\Gamma_{+},|v \cdot n|\right)}+\frac{1}{\varepsilon}\left\|u_{\varepsilon}-\int_{V} u_{\varepsilon}\right\|_{L^{2}(\Omega \times V)} \leq C\|q\|_{L^{2}(\Omega \times V)},
$$

where $L^{2}\left(\Gamma_{+},|v \cdot n|\right)$ is the trace space of functions u satisfying $\int_{\Gamma_{+}}(v \cdot n)|u|^{2} \mathrm{~d} \Gamma<\infty$ with $\Gamma_{+}=\{(x, v) \in$ $\partial \Omega \times V \mid v \cdot n(x)>0\}$ and $\mathrm{d} \Gamma=\mathrm{d} v \mathrm{~d} \sigma$ (d $\sigma$ being the surface measure on $\partial \Omega)$.

Proof. We multiply equation (21) by $u_{\varepsilon} \psi_{\varepsilon} \psi_{\varepsilon}^{*}$ and integrate to obtain

$$
I_{1}+I_{2}=\int_{\Omega} \int_{V} F_{\varepsilon}(q) \psi_{\varepsilon} \psi_{\varepsilon}^{*} u_{\varepsilon} \mathrm{d} x \mathrm{~d} v
$$

The first term is

$$
\begin{aligned}
I_{1} & =\frac{1}{\varepsilon} \int_{\Omega} \int_{V} v \cdot \nabla u_{\varepsilon}\left(u_{\varepsilon} \psi_{\varepsilon} \psi_{\varepsilon}^{*}\right) \mathrm{d} x \mathrm{~d} v=\frac{1}{\varepsilon} \int_{\Gamma_{+}}\left|u_{\varepsilon}\right|^{2} \psi_{\varepsilon} \psi_{\varepsilon}^{*} v \cdot n \mathrm{~d} v \mathrm{~d} \sigma \\
& -\frac{1}{\varepsilon} \int_{\Omega} \int_{V} v \cdot \nabla u_{\varepsilon}\left(u_{\varepsilon} \psi_{\varepsilon} \psi_{\varepsilon}^{*}\right) \mathrm{d} x \mathrm{~d} v-\frac{1}{\varepsilon} \int_{\Omega} \int_{V}\left|u_{\varepsilon}\right|^{2} v \cdot \nabla\left(\psi_{\varepsilon} \psi_{\varepsilon}^{*}\right) \mathrm{d} x \mathrm{~d} v .
\end{aligned}
$$

Multiplying the infinite medium equation (5) by $\psi^{*}$ and subtracting the adjoint equation (6) multiplied by $\psi$ yields

$$
v \cdot \nabla\left(\psi \psi^{*}\right)=\psi^{*} \int_{V} \psi\left(y, v^{\prime}\right) \sigma_{\infty}\left(y, v^{\prime}, v\right) \mathrm{d} v^{\prime}-\psi \int_{V} \psi^{*}\left(y, v^{\prime}\right) \sigma_{\infty}^{*}\left(y, v^{\prime}, v\right) \mathrm{d} v^{\prime}
$$

where $\sigma_{\infty}^{*}\left(y, v^{\prime}, v\right)=\sigma_{\infty}\left(y, v, v^{\prime}\right)$. Denoting by $\sigma_{\infty}^{\varepsilon *}\left(x, v^{\prime}, v\right)=\sigma_{\infty}^{\varepsilon}\left(x, v, v^{\prime}\right)$, we deduce that

$$
\frac{1}{\varepsilon} \int_{\Omega} \int_{V}\left|u_{\varepsilon}\right|^{2} v \cdot \nabla\left(\psi_{\varepsilon} \psi_{\varepsilon}^{*}\right) \mathrm{d} x \mathrm{~d} v=\frac{1}{\varepsilon^{2}} \int_{\Omega} \int_{V}\left|u_{\varepsilon}\right|^{2}\left(\psi_{\varepsilon}^{*} \int_{V} \psi_{\varepsilon} \sigma_{\infty}^{\varepsilon}-\psi_{\varepsilon} \int_{V} \psi_{\varepsilon}^{*} \sigma_{\infty}^{\varepsilon *}\right) \mathrm{d} x \mathrm{~d} v .
$$

This yields the following expression for the first integral $I_{1}$

$$
I_{1}=\frac{1}{2 \varepsilon} \int_{\Gamma_{+}}\left|u_{\varepsilon}\right|^{2} \psi_{\varepsilon} \psi_{\varepsilon}^{*} v \cdot n \mathrm{~d} v \mathrm{~d} \sigma-\frac{1}{2 \varepsilon^{2}} \int_{\Omega} \int_{V}\left|u_{\varepsilon}\right|^{2}\left(\psi_{\varepsilon}^{*} \int_{V} \psi_{\varepsilon} \sigma_{\infty}^{\varepsilon}-\psi_{\varepsilon} \int_{V} \psi_{\varepsilon}^{*} \sigma_{\infty}^{\varepsilon *}\right) \mathrm{d} x \mathrm{~d} v .
$$

The second term is given by

$$
\begin{aligned}
I_{2}=\frac{1}{\varepsilon} \int_{\Omega} \int_{V} Q_{\varepsilon}\left(u_{\varepsilon}\right)\left(u_{\varepsilon} \psi_{\varepsilon} \psi_{\varepsilon}^{*}\right) \mathrm{d} x \mathrm{~d} v= & \frac{1}{\varepsilon^{2}} \int_{\Omega} \int_{V}\left|u_{\varepsilon}\right|^{2} \psi_{\varepsilon}^{*}\left(\int_{V} \sigma_{\infty}^{\varepsilon} \psi_{\varepsilon}\right) \mathrm{d} x \mathrm{~d} v \\
& -\frac{1}{\varepsilon^{2}} \int_{\Omega} \int_{V} u_{\varepsilon} \psi_{\varepsilon}^{*}\left(\int_{V} \sigma_{\infty}^{\varepsilon} \psi_{\varepsilon} u_{\varepsilon}\right) \mathrm{d} x \mathrm{~d} v .
\end{aligned}
$$

Summing $I_{1}$ and $I_{2}$ we have

$$
\begin{aligned}
I_{1}+I_{2}= & \frac{1}{2 \varepsilon} \int_{\Gamma_{+}}\left|u_{\varepsilon}\right|^{2} \psi_{\varepsilon} \psi_{\varepsilon}^{*} v \cdot n \mathrm{~d} v \mathrm{~d} \sigma \\
& +\frac{1}{2 \varepsilon^{2}} \int_{\Omega} \int_{V}\left[\left|u_{\varepsilon}\right|^{2} \psi_{\varepsilon}^{*}\left(\int_{V} \sigma_{\infty}^{\varepsilon} \psi_{\varepsilon}\right)+\left|u_{\varepsilon}\right|^{2} \psi_{\varepsilon}\left(\int_{V} \sigma_{\infty}^{* \varepsilon} \psi_{\varepsilon}^{*}\right)-2 u_{\varepsilon} \psi_{\varepsilon}^{*}\left(\int_{V} \sigma_{\infty}^{\varepsilon} \psi_{\varepsilon} u_{\varepsilon}\right)\right] \mathrm{d} x \mathrm{~d} v,
\end{aligned}
$$

or equivalently

$$
\begin{aligned}
I_{1}+I_{2}= & \frac{1}{2 \varepsilon} \int_{\Gamma_{+}}\left|u_{\varepsilon}\right|^{2} \psi_{\varepsilon} \psi_{\varepsilon}^{*} v \cdot n \mathrm{~d} v \mathrm{~d} \sigma \\
& +\frac{1}{2 \varepsilon^{2}} \int_{\Omega} \int_{V} \int_{V} \sigma_{\infty}^{\varepsilon}\left(v^{\prime}, v\right) \psi_{\varepsilon}^{*}(v) \psi_{\varepsilon}\left(v^{\prime}\right)\left|u_{\varepsilon}(v)-u_{\varepsilon}\left(v^{\prime}\right)\right|^{2} \mathrm{~d} v \mathrm{~d} v^{\prime} \mathrm{d} x .
\end{aligned}
$$


From Proposition 2.5, we know that $\psi_{\varepsilon}^{*} \sigma_{\infty}^{\varepsilon} \psi_{\varepsilon}$ is bounded from below by a positive constant. On the other hand we easily check that

$$
\int_{\Omega} \int_{V} \int_{V}\left|u_{\varepsilon}(v)-u_{\varepsilon}\left(v^{\prime}\right)\right|^{2} \mathrm{~d} x \mathrm{~d} v \mathrm{~d} v^{\prime}=\int_{\Omega} \int_{V}\left|u_{\varepsilon}-\int_{V} u_{\varepsilon} \mathrm{d} v^{\prime}\right|^{2} \mathrm{~d} x \mathrm{~d} v .
$$

Therefore, we deduce the a priori estimate (recall that $v \cdot n>0$ on $\Gamma_{+}$)

$$
\frac{1}{\varepsilon}\left\|u_{\varepsilon}\right\|_{L^{2}\left(\Gamma_{+},|v \cdot n|\right)}^{2}+\frac{1}{\varepsilon^{2}}\left\|u_{\varepsilon}-\int_{V} u_{\varepsilon} \mathrm{d} v\right\|_{L^{2}(\Omega \times V)}^{2} \leq C\left\|u_{\varepsilon}\right\|_{L^{2}(\Omega \times V)}\|q\|_{L^{2}(\Omega \times V)} .
$$

On the other hand, equation (21) implies that

$$
\left\|v \cdot \nabla u_{\varepsilon}\right\|_{L^{2}(\Omega \times V)} \leq C\left[\varepsilon\|q\|_{L^{2}(\Omega \times V)}+\frac{1}{\varepsilon}\left\|Q_{\varepsilon}\left(u_{\varepsilon}\right)\right\|_{L^{2}(\Omega \times V)}\right] .
$$

From the properties of the scattering kernel $Q_{\varepsilon}$, we deduce that

$$
\left\|Q_{\varepsilon}\left(u_{\varepsilon}\right)\right\|_{L^{2}(\Omega \times V)}=\left\|Q_{\varepsilon}\left(u_{\varepsilon}-\int_{V} u_{\varepsilon} \mathrm{d} v\right)\right\|_{L^{2}(\Omega \times V)} \leq C\left\|u_{\varepsilon}-\int_{V} u_{\varepsilon} \mathrm{d} v\right\|_{L^{2}(\Omega \times V)} .
$$

Finally the Poincaré inequality in Lemma 4.3 gives

$$
\left\|u_{\varepsilon}\right\|_{L^{2}(\Omega \times V)} \leq C\left[\left\|u_{\varepsilon}\right\|_{L^{2}\left(\Gamma_{+},|v \cdot n|\right)}+\left\|v \cdot \nabla u_{\varepsilon}\right\|_{L^{2}(\Omega \times V)}\right] .
$$

A combination of inequalities $(25,26,27,28)$ yields the desired result.

Corollary 4.2. Let $q_{\varepsilon}$ be a bounded sequence in $L^{2}(\Omega \times V)$. Let $u_{\varepsilon}$ be the solution of (17) with the source term $q_{\varepsilon}$ instead of $q$. Then the sequence $u_{\varepsilon}$ is relatively compact in $L^{2}(\Omega \times V)$.

Proof. Remark that the energy estimate (24) holds true also for the source term $q_{\varepsilon}$. Therefore, $u_{\varepsilon}$ and $v \cdot \nabla u_{\varepsilon}$ are bounded in $L^{2}(\Omega \times V)$. By virtue of the averaging lemma [18], we deduce that $\int_{V} u_{\varepsilon} \mathrm{d} v$ is relatively compact. But (24) implies that $u_{\varepsilon}-\int_{V} u_{\varepsilon} \mathrm{d} v$ goes to 0 strongly in $L^{2}(\Omega \times V)$ which proves the relative compactness.

We now state a trace embedding result which is the analogue in transport theory of the Poincaré inequality for elliptic operators. Recall that, by assumptions (H1, H2), $\Omega$ is a bounded and convex open set in $\mathbb{R}^{N}$, and that $V$ is a compact set in $\mathbb{R}^{N}$ such that $0 \notin V$. Let us define the following maximum travel time of a particle (in the absence of collisions)

$$
\begin{array}{ll}
\tau(x, v)=\inf \{t>0, x-v t \notin V\}, & (x, v) \in \Gamma_{+} \\
\tau(x, v)=\inf \{t>0, x+v t \notin V\}, & (x, v) \in \Gamma_{-} .
\end{array}
$$

Remark that, since $\Omega$ is bounded, $\tau(x, v)$ is bounded independently of $(x, v) \in \partial \Omega \times V$. Let us also introduce the following measure on $\partial \Omega \times V$

$$
d \xi=|v \cdot n| \tau(x, v) \mathrm{d} v \mathrm{~d} \sigma
$$

where $\mathrm{d} \sigma$ is the surface measure on $\partial \Omega$

Lemma 4.3. Any function $u(x, v) \in W^{2}(\Omega \times V)$ has traces on $\Gamma_{+}$and $\Gamma_{-}$which belong to $L^{2}\left(\Gamma_{+}, d \xi\right)$ and $L^{2}\left(\Gamma_{-}, d \xi\right)$ respectively. Furthermore, there exists a constant $C$, independent of $u$, such that

$$
\|u\|_{L^{2}(\Omega \times V)}+\|u\|_{L^{2}\left(\Gamma_{+}, d \xi\right)} \leq C\left(\|v \cdot \nabla u\|_{L^{2}(\Omega \times V)}+\|u\|_{L^{2}\left(\Gamma_{-}, d \xi\right)}\right) .
$$


Proof. The first part of the lemma is simply the usual trace theorem in transport theory (see e.g. [14]). Thus it remains to prove inequality (30). For any smooth function $u(x, v) \in \Omega \times V$, we have

$$
u(x, v)=\int_{0}^{d(x, v)} v \cdot \nabla u(x-s v, v) \mathrm{d} s+u(\bar{x}, v),
$$

where $d(x, v)$ is the distance between $x \in \Omega$ and $\partial \Omega$ in the direction $-v$ and $\bar{x}$ the point in $\partial \Omega$ uniquely defined by

$$
\bar{x}=x-d(x, v) v .
$$

Since $\Omega$ is a bounded domain we deduce from the Cauchy-Schwartz inequality

$$
|u(x, v)|^{2} \leq C\left(\int_{0}^{d(x, v)}(v \cdot \nabla u)^{2}(x-s v, v) \mathrm{d} s+|u(\bar{x}, v)|^{2}\right) .
$$

Denoting by $\chi(x)$ the characteristic function of $\Omega$ (equal to 1 if $x \in \Omega$ and 0 otherwise), and integrating with respect to $x$ the first term in the right-hand side of (32) leads to

$$
\begin{aligned}
\int_{\Omega} \int_{0}^{d(x, v)}|v \cdot \nabla u|^{2}(x-s v, v) \mathrm{d} s \mathrm{~d} x & =\int_{\Omega} \int_{0}^{\operatorname{diam}(\Omega)} \chi(x-s v)|v \cdot \nabla u|^{2}(x-s v, v) \mathrm{d} s \mathrm{~d} x \\
& =\int_{0}^{\operatorname{diam}(\Omega)} \int_{\Omega+s v} \chi(y)|v \cdot \nabla u|^{2}(y, v) \mathrm{d} y \mathrm{~d} s \\
& =\int_{0}^{\operatorname{diam}(\Omega)} \int_{(\Omega+s v) \cap \Omega}|v \cdot \nabla u|^{2}(y, v) \mathrm{d} y \mathrm{~d} s \\
& \leq \operatorname{diam}(\Omega) \int_{\Omega}|v \cdot \nabla u|^{2}(y, v) \mathrm{d} y .
\end{aligned}
$$

We now integrate with respect to $x$ the second term in the right-hand side of (32). For a given velocity $v$ we introduce an orthonormal basis $\left(x_{1}, \ldots, x_{n}\right)=\left(x^{\prime}, x_{n}\right)$ such that $x_{n}$ is parallel to $v$. The boundary point $\bar{x}$, defined by (31), is thus a function of $x^{\prime}$ only. Then

$$
\int_{\Omega}|u(\bar{x}, v)|^{2} \mathrm{~d} x=\int_{\mathbb{R}^{N-1}}|u(\bar{x}, v)|^{2}\left(\int_{\mathbb{R}} \chi(x) \mathrm{d} x_{n}\right) \mathrm{d} x^{\prime}=\int_{\Omega^{\prime}}|u(\bar{x}, v)|^{2} \tau(\bar{x}, v) \mathrm{d} x^{\prime},
$$

where $\Omega^{\prime}$ is the projection of $\Omega$ on $\mathbb{R}^{N-1}$. Since $\Omega$ is convex, there is a diffeomorphism between $\Omega^{\prime}$ and that part of the boundary defined by $\Gamma_{v}^{-}=\{x \in \partial \Omega, n(x) \cdot v<0\}$. Changing the variable $x^{\prime}$ in $\bar{x} \in \Gamma_{v}^{-}$yields $\mathrm{d} x^{\prime}=|v \cdot n| \mathrm{d} \sigma$ and

$$
\int_{\Omega}|u(\bar{x}, v)|^{2} \mathrm{~d} x=\int_{\Gamma_{v}^{-}}|v \cdot n| \tau(\bar{x}, v)|u(\bar{x}, v)|^{2} \mathrm{~d} \sigma .
$$

Integrating over $V$ inequalities $(33,34)$ gives an upper bound for the norm of $u$ in $L^{2}(\Omega \times V)$. The same method can be applied to obtain an upper bound of the $L^{2}\left(\Gamma_{+}, d \xi\right)$-norm of $u$ in terms of the $L^{2}(\Omega \times V)$-norm of $u$ and $v \cdot \nabla u$, which yields the desired result.

\section{Convergence Proof}

This section is devoted to the proof of Theorem 3.6. We use the two-scale convergence method $[2,26]$. Let us remark that the more classical method of asymptotic expansions can also be used to prove this theorem $[7,8]$. However this is at the price of more assumptions on the smoothness of the physical data. 
Let us introduce some notations that we shall use in the derivation of the two-scale convergence properties. We denote by $C_{\#}^{\infty}(Y)$ the space of infinitely differentiable functions in $\mathbb{R}^{N}$ that are periodic of period $Y$, and $L_{\#}^{2}(Y)$ (respectively, $H_{\#}^{1}(Y)$ ) the completion of $C_{\#}^{\infty}(Y)$ for the norm of $L^{2}(Y)$ (respectively, $H^{1}(Y)$ ). Generalizing the usual definition of two-scale convergence to functions that depend on two variables $x$ and $v$, we introduce the following

Definition 5.1. A sequence of functions $u_{\varepsilon}$ in $L^{2}(\Omega \times V)$ is said to two-scale converge to a limit $u^{0}(x, y, v)$ in $L^{2}(\Omega \times Y \times V)$ if, for any function $\psi(x, y, v)$ in $\mathcal{D}\left[\Omega \times V ; C_{\#}^{\infty}(Y)\right]$, we have

$$
\lim _{\varepsilon \rightarrow 0} \int_{\Omega} \int_{V} u_{\varepsilon}(x, v) \psi(x, x / \varepsilon, v) \mathrm{d} x \mathrm{~d} v=\int_{\Omega} \int_{Y} \int_{V} u^{0}(x, y, v) \psi(x, y, v) \mathrm{d} x \mathrm{~d} y \mathrm{~d} v .
$$

This definition makes sense because of the following compactness theorem (the proof of which is a straightforward adaptation of Lem. 1.3 and Th. 1.2 in [2]).

Theorem 5.2. From each bounded sequence $u_{\varepsilon}$ in $L^{2}(\Omega \times V)$ we can extract a subsequence, and there exists a limit $u^{0}(x, y, v)$ in $L^{2}(\Omega \times Y \times V)$ such that this subsequence two-scale converges to $u^{0}$.

We now prove a result which is the analogue of Proposition 1.14 in [2] for transport theory.

Proposition 5.3. Let $u_{\varepsilon}$ be a sequence in $L^{2}(\Omega \times V)$ such that there exists a constant $C$, independent of $\varepsilon$, satisfying

$$
\begin{array}{ll}
\left\|u_{\varepsilon}\right\|_{L^{2}(\Omega \times V)}+\left\|v \cdot \nabla u_{\varepsilon}\right\|_{L^{2}(\Omega \times V)} \leq C, & u_{\varepsilon}=0 \quad \text { on } \Gamma_{-}, \\
\left\|u_{\varepsilon}\right\|_{L^{2}\left(\Gamma_{+},|v \cdot n|\right)} \leq C \sqrt{\varepsilon}, & \left\|u_{\varepsilon}-\int_{V} u_{\varepsilon} \mathrm{d} v\right\|_{L^{2}(\Omega \times V)} \leq C \varepsilon .
\end{array}
$$

Then, there exists $u^{0}(x)$ in $H_{0}^{1}(\Omega)$ and $u^{1}(x, y, v)$ in $L^{2}\left(\Omega \times V ; H_{\#}^{1}(Y)\right)$ such that, up to the extraction of subsequences, $u_{\varepsilon}(x, v)$ converges strongly to $u^{0}(x)$ in $L^{2}(\Omega \times V), v \cdot \nabla u_{\varepsilon}(x, v)$ two-scale converges to $v \cdot \nabla_{x} u^{0}+$ $v \cdot \nabla_{y} u^{1}$, and $\varepsilon^{-1}\left(u_{\varepsilon}-\int_{V} u_{\varepsilon} \mathrm{d} v\right)$ two-scale converges to $\left(u^{1}-\int_{V} u^{1} \mathrm{~d} v\right)$.

Proof. Since $u_{\varepsilon}$ is bounded in $L^{2}(\Omega \times V)$, Theorem 5.2 implies the existence of $u^{0}(x, y, v) \in L^{2}(\Omega \times Y \times V)$ such that, up to a subsequence, $u_{\varepsilon}$ two-scale converges to $u^{0}$. Since $\left\|u_{\varepsilon}-\int_{V} u_{\varepsilon} \mathrm{d} v\right\| \leq C \varepsilon$ we deduce that

$$
u^{0}(x, y, v)=\int_{V} u^{0}(x, y, v) \mathrm{d} v .
$$

On the other hand, $v \cdot \nabla u_{\varepsilon}$ being bounded in $L^{2}(\Omega \times V)$, for any smooth $Y$-periodic function $\psi(x, y, v)$ we have

$$
\lim _{\varepsilon \rightarrow 0} \varepsilon \int_{\Omega} \int_{V} v \cdot \nabla u_{\varepsilon} \psi(x, x / \varepsilon, v) \mathrm{d} x \mathrm{~d} v=\lim _{\varepsilon \rightarrow 0}-\int_{\Omega} \int_{V} u_{\varepsilon} v \cdot \nabla_{y} \psi(x, x / \varepsilon, v) \mathrm{d} x \mathrm{~d} v=0,
$$

which implies in the limit as $\varepsilon$ goes to zero that

$$
\int_{\Omega} \int_{Y} \int_{V} u^{0}(x, y) v \cdot \nabla_{y} \psi(x, y, v) \mathrm{d} x \mathrm{~d} y \mathrm{~d} v=0 .
$$

Consequently we have $v \cdot \nabla_{y} u^{0}(x, y)=0$. Since $V$ contains at least a basis of $\mathbb{R}^{N}$ (this is clearly true for a velocity space of unit measure and for an union of spheres), we easily deduce that

$$
u^{0}(x, y) \equiv u^{0}(x)
$$

To prove the strong convergence of $u_{\varepsilon}$, we use the compactness result of the averaging lemma in [18] which states that, $u_{\varepsilon}$ and $v \cdot \nabla u_{\varepsilon}$ being bounded in $L^{2}(\Omega \times V), \int_{V} u_{\varepsilon}(x, v) \mathrm{d} v$ is relatively compact in $L^{2}(\Omega)$. By assumption $\left\|u_{\varepsilon}-\int_{V} u_{\varepsilon}(x, v) \mathrm{d} v\right\|_{L^{2}(\Omega \times V)} \leq C \varepsilon$ which implies that $u_{\varepsilon}$ converges strongly to $u^{0}$ in $L^{2}(\Omega \times V)$. 
Next, we focus on the two scale convergence of $v \cdot \nabla u_{\varepsilon}$. Since it is a bounded sequence in $L^{2}(\Omega \times V)$, by virtue of Theorem 5.2 there exists $\xi(x, y, v) \in L^{2}(\Omega \times Y \times V)$ such that, up to a subsequence, $v \cdot \nabla u_{\varepsilon}$ two-scale converges to $\xi$. Denoting by $L_{\#}^{2}(\Omega \times Y \times V)$ the space of $Y$-periodic functions in $L^{2}(\Omega \times Y \times V)$, let us define its subspace

$$
H=\left\{\psi \in L_{\#}^{2}(\Omega \times Y \times V) \text { s.t. } v \cdot \nabla_{y} \psi=0\right\} .
$$

Clearly $H$ is a closed subspace of $L_{\#}^{2}(\Omega \times Y \times V)$ and, by Fourier analysis, its orthogonal is easily seen to be

$$
H^{\perp}=\left\{\phi \in L_{\#}^{2}(\Omega \times Y \times V) \text { s.t. } \exists \psi \in L_{\#}^{2}(\Omega \times Y \times V), \phi=v \cdot \nabla_{y} \psi\right\} .
$$

For a smooth function $\phi \in H$, having compact support in $\Omega$, we have

$$
\int_{\Omega} \int_{V} v \cdot \nabla u_{\varepsilon} \phi\left(x, \frac{x}{\varepsilon}, v\right) \mathrm{d} x \mathrm{~d} v=-\int_{\Omega} \int_{V} u_{\varepsilon} v \cdot \nabla_{x} \phi\left(x, \frac{x}{\varepsilon}, v\right) \mathrm{d} x \mathrm{~d} v .
$$

Passing to the two-scale limit and integrating by parts we get

$$
\int_{\Omega} \int_{Y} \int_{V}\left[\xi(x, y, v)-v \cdot \nabla_{x} u^{0}(x)\right] \phi(x, y, v) \mathrm{d} x \mathrm{~d} y \mathrm{~d} v=0 .
$$

By density (37) holds true for any $\phi \in H$. Choosing $\phi=\phi(x, v)$ implies that $v \cdot \nabla_{x} u^{0}=\int_{Y} \xi \mathrm{d} y$ and therefore $v \cdot \nabla_{x} u^{0}(x)$ belongs to $L^{2}(\Omega \times V)$, which in turn implies $u^{0} \in H^{1}(\Omega)$. Eventually, (37) is equivalent to $\left[\xi(x, y, v)-v \cdot \nabla_{x} u^{0}(x)\right] \in H^{\perp}$. Thus there exists a (non-unique) function $\tilde{u}^{1}(x, y, v) \in L_{\#}^{2}(\Omega \times Y \times V)$ such that

$$
\xi(x, y, v)=v \cdot \nabla_{x} u^{0}(x)+v \cdot \nabla_{y} \tilde{u}^{1}(x, y, v) .
$$

Finally we show that $u^{0}$ vanishes on the boundary $\partial \Omega$. Consider a smooth function $\phi(x, v)$. Since $u_{\varepsilon}=0$ on $\Gamma_{-}$an integrations by parts yields

$$
\int_{\Omega} \int_{V} v \cdot \nabla u_{\varepsilon} \phi \mathrm{d} x \mathrm{~d} v=-\int_{\Omega} \int_{V} u_{\varepsilon} v \cdot \nabla \phi \mathrm{d} x \mathrm{~d} v+\int_{\Gamma_{+}} u_{\varepsilon}(s, v) \phi(s, v) v \cdot n \mathrm{~d} v \mathrm{~d} \sigma .
$$

Since $\left\|u_{\varepsilon}\right\|_{L^{2}\left(\Gamma^{+},|v \cdot n|\right)} \leq C \sqrt{\varepsilon}$, passing to the limit leads to

$$
\int_{\Omega} \int_{Y} \int_{V}\left(v \cdot \nabla_{x} u^{0}+v \cdot \nabla_{y} \tilde{u}^{1}\right) \phi \mathrm{d} x \mathrm{~d} y \mathrm{~d} v=-\int_{\Omega} \int_{Y} \int_{V} u^{0} v \cdot \nabla_{x} \phi \mathrm{d} x \mathrm{~d} y \mathrm{~d} v .
$$

By periodicity $\int_{Y} v \cdot \nabla_{y} \tilde{u}^{1} \mathrm{~d} y=0$, and therefore integrating by parts gives

$$
\int_{\Gamma_{+}} \phi(x, v) u^{0}(x) v \cdot n \mathrm{~d} v \mathrm{~d} \sigma=0
$$

for any smooth function $\phi(x, v)$, which implies $u^{0}(x)=0$ on $\partial \Omega$.

Next, we study the two-scale convergence of $w_{\varepsilon}=\varepsilon^{-1}\left(u_{\varepsilon}-\int_{V} u_{\varepsilon}\right)$. Since, by assumption, it is a bounded sequence in $L^{2}(\Omega \times V)$, there exists a function $w(x, y, v) \in L^{2}(\Omega \times Y \times V)$ such that, up to a subsequence, $w_{\varepsilon}$ two-scale converges to $w(x, y, v)$. Let us introduce the space

$$
G=\left\{\phi \in L_{\#}^{2}(\Omega \times Y \times V) \text { s.t. } \int_{V} v \cdot \nabla_{y} \phi(x, y, v) \mathrm{d} v=0\right\}
$$


It is a closed subspace of $L_{\#}^{2}(\Omega \times Y \times V)$ and, by Fourier analysis, one can check that its orthogonal is

$$
G^{\perp}=\left\{\phi \in L_{\#}^{2}(\Omega \times Y \times V) \text { s.t. } \exists \theta(x, y) \in L_{\#}^{2}(\Omega \times Y) \phi=v \cdot \nabla_{y} \theta\right\} .
$$

Let $\phi(x, y, v)$ be a smooth function in $G$ with compact support in $\Omega$. Then

$$
\varepsilon \int_{\Omega} \int_{V} w_{\varepsilon} v \cdot \nabla(\phi(x, x / \varepsilon, v)) \mathrm{d} x \mathrm{~d} v=-\varepsilon \int_{\Omega} \int_{V} \phi(x, x / \varepsilon, v) v \cdot \nabla w_{\varepsilon} \mathrm{d} x \mathrm{~d} v
$$

or equivalently

$$
\begin{aligned}
\int_{\Omega} \int_{V} w_{\varepsilon}\left(v \cdot \nabla_{y} \phi+\varepsilon v \cdot \nabla_{x} \phi\right) \mathrm{d} x \mathrm{~d} v & =-\int_{\Omega} \int_{V} \phi v \cdot \nabla u_{\varepsilon} \mathrm{d} x \mathrm{~d} v+\int_{\Omega} \int_{V} \phi v \cdot \nabla\left(\int_{V} u_{\varepsilon} \mathrm{d} v\right) \mathrm{d} x \mathrm{~d} v \\
& =-\int_{\Omega} \int_{V} \phi v \cdot \nabla u_{\varepsilon} \mathrm{d} x \mathrm{~d} v-\int_{\Omega} \int_{V}\left(\int_{V} u_{\varepsilon} \mathrm{d} v\right)\left(v \cdot \nabla_{x} \phi+\frac{1}{\varepsilon} v \cdot \nabla_{y} \phi\right) \mathrm{d} x \mathrm{~d} v \\
& =-\int_{\Omega} \int_{V} \phi v \cdot \nabla u_{\varepsilon} \mathrm{d} x \mathrm{~d} v-\int_{\Omega} \int_{V}\left(\int_{V} u_{\varepsilon} \mathrm{d} v\right) v \cdot \nabla_{x} \phi \mathrm{d} x \mathrm{~d} v .
\end{aligned}
$$

Passing to the two-scale limit yields

$$
\int_{\Omega} \int_{Y} \int_{V} w v \cdot \nabla_{y} \phi \mathrm{d} x \mathrm{~d} y \mathrm{~d} v=-\int_{\Omega} \int_{Y} \int_{V} \phi\left(v \cdot \nabla_{x} u^{0}+v \cdot \nabla_{y} \tilde{u}^{1}\right) \mathrm{d} x \mathrm{~d} y \mathrm{~d} v-\int_{\Omega} \int_{Y} \int_{V} u^{0} v \cdot \nabla_{x} \phi \mathrm{d} x \mathrm{~d} y \mathrm{~d} v .
$$

Integrating by parts, we deduce that, for any $\phi \in G$,

$$
\int_{\Omega} \int_{Y} \int_{V}\left(-v \cdot \nabla_{y} w+v \cdot \nabla_{y} \tilde{u}^{1}\right) \phi \mathrm{d} x \mathrm{~d} y \mathrm{~d} v=0 .
$$

Therefore $v \cdot \nabla_{y}\left(\tilde{u}^{1}-w\right)$ belongs to $G^{\perp}$ and there exists a function $\theta(x, y) \in L_{\#}^{2}(\Omega \times Y)$ such that $v \cdot \nabla_{y}(w-$ $\left.\tilde{u}^{1}-\theta\right)=0$. In other words we have just proved that

$$
w(x, y, v)=\tilde{u}^{1}(x, y, v)+\theta(x, y)+\eta(x, y, v) \text { with } \eta \in L_{\#}^{2}(\Omega \times Y \times V), v \cdot \nabla_{y} \eta=0 .
$$

Recall that the function $\tilde{u}^{1}$, deduced from (37), is not unique. Consequently, introducing $u^{1}=\tilde{u}^{1}+\eta$, the sequence $v \cdot \nabla u_{\varepsilon}$ still two-scale converges to $v \cdot \nabla_{x} u^{0}+v \cdot \nabla_{y} u^{1}$, and furthermore we have

$$
w(x, y, v)=u^{1}(x, y, v)+\theta(x, y) .
$$

By definition $\int_{V} w_{\varepsilon}(x, v) \mathrm{d} v=0$, so that $\int_{V} w(x, y, v) \mathrm{d} v=0$. This implies that

$$
\theta(x, y)=-\int_{V} u^{1}(x, y, v) \mathrm{d} v,
$$

which concludes the proof.

We turn to the homogenization of problem (21). From now on, $u_{\varepsilon}$ denotes the unique solution of (21) and $u^{0}, u^{1}$ are the associated two-scale limits given by Proposition 5.3 (up to a subsequence). Recall that $\psi$ and $\psi^{*}$ are the solutions of the infinite medium problems (5) and (6) respectively. 
Proposition 5.4. If the drift flux $J=\int_{Y} \int_{V} v \psi \psi^{*} \mathrm{~d} v \mathrm{~d} y$ is non-zero, then $u^{0}(x)=0$ and the entire sequence $u_{\varepsilon}$ converges strongly to 0 in $L^{2}(\Omega \times V)$. If $J=0$, then $u^{1}$ and $u^{0}$ satisfy

$$
u^{1}(x, y, v)=\sum_{j=1}^{N} \frac{\partial u^{0}}{\partial x_{j}}(x) \theta^{j}(y, v),
$$

where $\theta^{j}$ is the unique solution of (16) up to an additive constant.

Proof. Let $\phi(x, y, v)$ be a smooth $Y$-periodic function. Multiplying (21) by $\varepsilon \phi(x, x / \varepsilon, v)$ yields

$$
\int_{\Omega} \int_{V} v \cdot \nabla u_{\varepsilon} \phi \mathrm{d} x \mathrm{~d} v+\varepsilon^{-1} \int_{\Omega} \int_{V} Q_{\varepsilon}\left(u_{\varepsilon}\right) \phi \mathrm{d} x \mathrm{~d} v=\int_{\Omega} \int_{V} \varepsilon \phi F_{\varepsilon}(q) .
$$

Recalling that $\varepsilon^{-1} Q_{\varepsilon}\left(u_{\varepsilon}\right)=Q_{\varepsilon}\left(w_{\varepsilon}\right)$ (see Rem. 3.5), and introducing the adjoint scattering kernel $Q_{\varepsilon}^{*}$, we obtain

$$
\varepsilon^{-1} \int_{\Omega} \int_{V} Q_{\varepsilon}\left(u_{\varepsilon}\right) \phi \mathrm{d} x \mathrm{~d} v=\int_{\Omega} \int_{V} w_{\varepsilon} Q_{\varepsilon}^{*}(\phi) \mathrm{d} x \mathrm{~d} v
$$

We easily check that $Q_{\varepsilon}^{*}(\phi(x, x / \varepsilon, v))$ two-scale converges to $Q^{*}(\phi)$, and furthermore that

$$
\lim _{\varepsilon \rightarrow 0}\left\|Q_{\varepsilon}^{*}(\phi(x, x / \varepsilon, v))\right\|_{L^{2}(\Omega \times V)}=\left\|Q^{*}(\phi(x, y, v))\right\|_{L^{2}(\Omega \times Y \times V)}
$$

which is precisely the condition for passing to the two-scale limit in a product of two weakly converging sequences (see Th. 1.8 in $[2]$ )

$$
\lim _{\varepsilon \rightarrow 0} \int_{\Omega} \int_{V} w_{\varepsilon} Q_{\varepsilon}^{*}(\phi) \mathrm{d} x \mathrm{~d} v=\int_{\Omega} \int_{Y} \int_{V}\left(u^{1}-\int_{V} u^{1} \mathrm{~d} v^{\prime}\right) Q^{*}(\phi) \mathrm{d} x \mathrm{~d} y \mathrm{~d} v .
$$

As a consequence we obtain

$$
\int_{\Omega} \int_{Y} \int_{V}\left(v \cdot \nabla_{x} u^{0}+v \cdot \nabla_{y} u^{1}\right) \phi \mathrm{d} x \mathrm{~d} y \mathrm{~d} v+\int_{\Omega} \int_{Y} \int_{V} Q\left(u^{1}-\int_{V} u^{1} \mathrm{~d} v^{\prime}\right) \phi \mathrm{d} x \mathrm{~d} y \mathrm{~d} v=0,
$$

which implies that $u^{1}$ is a solution of

$$
v \cdot \nabla_{y} u^{1}+Q\left(u^{1}\right)=-v \cdot \nabla_{x} u^{0}
$$

Since $u^{0}$ does not depend on $y$ and $v$, we have $u^{1}=\sum_{j=1}^{N} \frac{\partial u^{0}}{\partial x_{j}} \theta^{j}$ where each $\theta^{j}(y, v)$ is a solution of

$$
\left\{\begin{array}{l}
v \cdot \nabla \theta^{j}+Q\left(\theta^{j}\right)=-v_{j} \quad \text { in } Y \times V \\
y \rightarrow \theta^{j}(y, v) Y-\text { periodic. }
\end{array}\right.
$$

The existence theory for (38) can be addressed directly, but it is simpler to remark that the product $\chi^{j}=\psi \theta^{j}$ is a solution of

$$
\left\{\begin{array}{l}
v \cdot \nabla \chi^{j}+\Sigma \chi^{j}=\int_{V} f \chi^{j} \mathrm{~d} v^{\prime}+\lambda_{\infty} \int_{V} \sigma \chi^{j} \mathrm{~d} v^{\prime}-v_{j} \psi \\
y \rightarrow \chi^{j}(y, v) Y \text { - periodic }
\end{array}\right.
$$

for which Proposition 2.4 applies. It states that there exists a solution $\chi^{j}$ if and only if the j-th component of the drift-flux is $J_{j}=0$, and it is unique up to the addition of a multiple of $\psi$. Therefore, either $J_{j}=\int_{Y} \int_{V} v_{j} \psi \psi^{*}=0$ and there exists a unique solution $\theta^{j}$ (up to an additive constant) of (38), or $J_{j} \neq 0$ and there is no solution except 0 if the right-hand side is 0 , which implies that $\partial u^{0} / \partial x_{j}=0$, i.e. $u^{0}=0$ due to the boundary condition. 
Before deriving the main convergence result of this paper, we prove that the postulated homogenized diffusion matrix is actually positive definite. This is the aim of the following lemma.

Lemma 5.5. The diffusion tensor $D=\left(D_{i j}\right)_{1 \leq i, j \leq N}$, defined by

$$
D_{i j}=-\int_{Y} \int_{V} v_{j} \psi(y, v) \psi^{*}(y, v) \theta^{i}(y, v) \mathrm{d} v \mathrm{~d} y
$$

is positive definite.

Proof. Let $\xi \in \mathbb{R}^{N}$ and $\theta_{\xi}=\sum_{i=1}^{N} \xi_{i} \theta^{i}$. Multiplying the cell equation satisfied by $\theta_{\xi}$ by $\theta_{\xi} \psi \psi^{*}$, a computation similar to that of Lemma 4.1 yields

$$
\begin{aligned}
D \xi \cdot \xi & =\int_{Y} \int_{V}\left(v \cdot \nabla_{y} \theta_{\xi}+Q\left(\theta_{\xi}\right)\right) \theta_{\xi} \psi \psi^{*} \mathrm{~d} y \mathrm{~d} v \\
& =\frac{1}{2} \int_{Y} \int_{V^{\prime}} \int_{V} \sigma_{\infty} \psi^{*}(v) \psi\left(v^{\prime}\right)\left|\theta_{\xi}(v)-\theta_{\xi}\left(v^{\prime}\right)\right|^{2} \mathrm{~d} y \mathrm{~d} v \mathrm{~d} v^{\prime} .
\end{aligned}
$$

This implies that $D \xi \cdot \xi \geq 0$ for all $\xi \in \mathbb{R}^{N}$. Assume now that $D \xi \cdot \xi=0$. It implies that $\theta_{\xi}$ does not depend on $v$, and thus $Q\left(\theta_{\xi}\right)=0$. This yields

$$
v \cdot \nabla_{y} \theta_{\xi}=v \cdot \xi \quad \text { in } Y \times V
$$

Since $V$ contains $N$ independent directions and $\theta_{\xi}$ does not depend on $v$, we conclude that $\nabla_{y} \theta_{\xi}=-\xi$ which is not possible for a $Y$-periodic function except if $\xi=0$. This proves that there exists a positive constant $C$ such that $D \xi \cdot \xi \geq C|\xi|^{2}$.

Remark 5.6. The homogenized diffusion matrix $D$ defined in Lemma 5.5 is not necessarily symmetric. However only its symmetric part plays a role in the homogenized equation (22) since it can easily be checked that adding to it a constant skew-symmetric matrix does not change the solution of the diffusion equation.

After these preliminaries we are in a position to prove Theorem 3.6. For the reader's convenience, we recall it here.

Theorem 5.7. If $J=0$, then the entire sequence $u_{\varepsilon}$ converges to $u^{0}(x)$ in $L^{2}(\Omega \times V)$, which is the unique solution in $H_{0}^{1}(\Omega)$ of

$$
\begin{cases}-\nabla \cdot D \nabla u(x)=F(q)(x) & \text { in } \Omega \\ u(x)=0 & \text { on } \partial \Omega\end{cases}
$$

where the diffusion tensor $D$ is defined as in Lemma 5.5, and $F(q) \in L^{2}(\Omega)$ is given by

$$
F(q)(x)=\int_{Y} \int_{V} \int_{V} \sigma\left(y, v^{\prime}, v\right) \psi^{*}\left(y, v^{\prime}\right) \psi(y, v) q(x, v) \mathrm{d} v \mathrm{~d} v^{\prime} \mathrm{d} y .
$$

Proof. Let us first define, for $1 \leq i \leq N$, the adjoint equation of the cell problem (38)

$$
\left\{\begin{array}{l}
-v \cdot \nabla_{y}\left(\psi \psi^{*} \theta_{i}^{*}\right)+Q^{*}\left(\psi \psi^{*} \theta_{i}^{*}\right)=v_{i} \psi \psi^{*} \quad \text { in } Y \times V \\
y \rightarrow \theta_{i}^{*}(y, v) Y \text {-periodic }
\end{array}\right.
$$


which admits a unique solution, up to an additive constant, since $\int_{Y} \int_{V} v_{i} \psi \psi^{*} \mathrm{~d} y \mathrm{~d} v=0$. Let $\phi(x)$ be a smooth function with compact support in $\Omega$. We define

$$
\phi^{1}(x, y, v)=\sum_{i=1}^{N} \frac{\partial \phi}{\partial x_{i}}(x) \theta_{i}^{*}(y, v)
$$

and

$$
\phi^{\varepsilon}(x, v)=\phi(x)+\varepsilon \phi^{1}(x, x / \varepsilon, v) .
$$

Remark that the test function $\phi^{\varepsilon}$ has the same structure as the two-scale limit of $u_{\varepsilon}$ but adjoint (this is an usual rule of thumb for constructing test functions in homogenization). Multiplying equation (21) by $\phi^{\varepsilon} \psi_{\varepsilon} \psi_{\varepsilon}^{*}$ and integrating by parts yields

$$
-\frac{1}{\varepsilon} \int_{\Omega} \int_{V} u_{\varepsilon} v \cdot \nabla\left(\phi^{\varepsilon} \psi_{\varepsilon} \psi_{\varepsilon}^{*}\right)+\frac{1}{\varepsilon^{2}} \int_{\Omega} \int_{V} u_{\varepsilon} Q_{\varepsilon}^{*}\left(\phi^{\varepsilon} \psi_{\varepsilon} \psi_{\varepsilon}^{*}\right)=\int_{\Omega} \int_{V} \phi^{\varepsilon} \psi_{\varepsilon} \psi_{\varepsilon}^{*} F_{\varepsilon}(q) .
$$

The first term in (40) becomes

$$
\begin{aligned}
& K_{1}=\int_{\Omega} \int_{V} {\left[-\frac{1}{\varepsilon} u_{\varepsilon} v \cdot \nabla\left(\phi \psi_{\varepsilon} \psi_{\varepsilon}^{*}\right)-u_{\varepsilon} v \cdot \nabla\left(\phi^{1} \psi_{\varepsilon} \psi_{\varepsilon}^{*}\right)\right] } \\
&=\int_{\Omega} \int_{V}\left[-\frac{1}{\varepsilon} u_{\varepsilon} \phi v \cdot \nabla\left(\psi_{\varepsilon} \psi_{\varepsilon}^{*}\right)-\frac{1}{\varepsilon} u_{\varepsilon} \psi_{\varepsilon} \psi_{\varepsilon}^{*} v \cdot \nabla_{x} \phi-\frac{1}{\varepsilon} u_{\varepsilon} v \cdot \nabla_{y}\left(\phi^{1} \psi \psi^{*}\right)(x, x / \varepsilon)\right. \\
&\left.\quad-u_{\varepsilon} \psi_{\varepsilon} \psi_{\varepsilon}^{*} v \cdot \nabla_{x}\left(\phi^{1}\right)(x, x / \varepsilon)\right],
\end{aligned}
$$

since $\psi$ and $\psi^{*}$ depend only on $y$, while $\phi$ depends only on $x$. On the other hand, the second term in (40) becomes

$$
K_{2}=\frac{1}{\varepsilon^{2}} \int_{\Omega} \int_{V} u_{\varepsilon} \phi Q_{\varepsilon}^{*}\left(\psi_{\varepsilon} \psi_{\varepsilon}^{*}\right)+\frac{1}{\varepsilon} \int_{\Omega} \int_{V} u_{\varepsilon} Q_{\varepsilon}^{*}\left(\psi_{\varepsilon} \psi_{\varepsilon}^{*} \phi^{1}\right)
$$

because, for $T=Q_{\varepsilon}$ or $Q_{\varepsilon}^{*}, T(\phi(x) u(x, v))=\phi(x) T(u(x, v))$ (see Rem. 3.5 on the properties of the scattering kernel $\left.Q_{\varepsilon}\right)$. Recalling that $\psi_{\varepsilon} \psi_{\varepsilon}^{*}$ satisfies the adjoint equation

$$
-\frac{1}{\varepsilon} v \cdot \nabla\left(\psi_{\varepsilon} \psi_{\varepsilon}^{*}\right)+\frac{1}{\varepsilon^{2}} Q_{\varepsilon}^{*}\left(\psi_{\varepsilon} \psi_{\varepsilon}^{*}\right)=0,
$$

we deduce that, when adding $K_{1}$ and $K_{2}$, the first terms in the right-hand sides of (41) and (42) cancel out. Furthermore, in view of the definition of $\phi^{1}$ and of equation (39) satisfied by $\theta_{i}^{*}$, we have

$$
-v \cdot \nabla_{y}\left(\psi \psi^{*} \phi^{1}\right)+Q^{*}\left(\psi \psi^{*} \phi^{1}\right)=\psi \psi^{*} v \cdot \nabla_{x} \phi .
$$

Therefore, when adding $K_{1}$ and $K_{2}$, the second and third terms in the right-hand side of (41) cancel out with the second term in the right-hand side of (42). Eventually, we get

$$
K_{1}+K_{2}=-\int_{\Omega} \int_{V} u_{\varepsilon} \psi_{\varepsilon} \psi_{\varepsilon}^{*} v \cdot \nabla_{x} \phi^{1}=-\sum_{i, j=1}^{N} \int_{\Omega} \int_{V} u_{\varepsilon} \psi_{\varepsilon} \psi_{\varepsilon}^{*} v_{j} \frac{\partial^{2} \phi}{\partial x_{i} \partial x_{j}}(x) \theta_{i}^{*}(x / \varepsilon, v) .
$$


Since $u_{\varepsilon}(x, v)$ converges strongly to $u^{0}(x)$ in $L^{2}(\Omega \times V)$ we can pass to the limit as $\varepsilon$ goes to 0

$$
-\sum_{i, j=1}^{N} \int_{\Omega} u^{0}(x) \frac{\partial^{2} \phi}{\partial x_{i} \partial x_{j}}(x)\left(\int_{Y} \int_{V} \psi \psi^{*} v_{j} \theta_{i}^{*}(y, v) \mathrm{d} y \mathrm{~d} v\right) \mathrm{d} x=\int_{\Omega} F(q) \mathrm{d} x .
$$

Multiplying (39) by $\theta^{j}$ and integrating by parts, we easily obtain that

$$
\int_{Y} \int_{V} v_{i} \psi \psi^{*} \theta^{j} \mathrm{~d} y \mathrm{~d} v=-\int_{Y} \int_{V} v_{j} \psi \psi^{*} \theta_{i}^{*}(y, v) \mathrm{d} y \mathrm{~d} v=-D_{i j} .
$$

This yields, for any smooth function $\phi(x)$,

$$
-\int_{\Omega} u^{0}(x) \sum_{i, j=1}^{N} D_{i j} \frac{\partial^{2} \phi}{\partial x_{i} \partial x_{j}}(x) \mathrm{d} x=\int_{\Omega} \phi(x) F(q)(x) \mathrm{d} x
$$

which is nothing but the homogenized equation. Since it admits a unique solution $u^{0}$ in $H_{0}^{1}(\Omega)$, all converging subsequences of $u_{\varepsilon}$ have the same limit, which proves that the entire sequence $u_{\varepsilon}$ converges to $u^{0}$.

\section{Correctors And numerical Result}

In the previous sections, we gave the asymptotic limit of the spectral problem under very mild smoothness hypothesis for both the cross sections and the solutions. Basically we have only assumed that the criticality problem was well posed for any $\varepsilon$ and that the solution of the infinite medium problem was bounded. With more smoothness available, one can expect some stronger convergence properties, namely so-called corrector results. This is a classical matter in homogenization theory for diffusion equations (see e.g. [28]) where correctors for the eigenvalues and eigenvectors can be obtained under additional assumptions. The question of correctors is more delicate in transport theory because it is intimately linked with the emergence of boundary layers. We shall not dwell on such problems here and we refer to $[7,8]$ for more details (a few comments on this issue are given in our discussion on the numerical application of homogenization at the end of this section). However, staying in the framework of the two-scale convergence method, we can obtain a partial corrector result for the eigenvectors. It gives a rate of convergence of the neutron flux at the boundary as well as a corrector for the deviation of the neutron flux from its velocity average. Unfortunately, it does not imply any corrector for the eigenvalues.

Theorem 6.1. Assume that the drift flux satisfies $J=0$. Let $u_{\varepsilon}$ be the unique solution of (21). Let $u^{0}$ be the unique solution of the homogenized equation (22), and define $u^{1}$ by

$$
u^{1}(x, y, v)=\sum_{j=1}^{N} \frac{\partial u^{0}}{\partial x_{j}}(x) \theta^{j}(y, v)
$$

where $\theta^{j}$ is the unique solution of the cell problem (16). Assume that $u^{0}(x)$ is smooth (say continuous in $\Omega$ ). Then

and

$$
\lim _{\varepsilon \rightarrow 0} \frac{1}{\varepsilon} \int_{\Gamma_{+}}\left|u_{\varepsilon}\right|^{2}|v \cdot n| \mathrm{d} \Gamma=0
$$

$$
\lim _{\varepsilon \rightarrow 0} \frac{1}{\varepsilon}\left\|\left(u_{\varepsilon}-\int_{V} u_{\varepsilon} \mathrm{d} v\right)-\varepsilon w_{\varepsilon}\right\|_{L^{2}(\Omega \times V)}=0
$$

with $w_{\varepsilon}(x, v)=\left(u^{1}(x, x / \varepsilon, v)-\int_{V} u^{1}(x, x / \varepsilon, v) \mathrm{d} v\right)$. 
Remark 6.2. Theorem 6.1 applies for the solutions of the source problem (21). However, since the eigenvectors of the spectral problem (17) converge strongly in $L^{2}(\Omega \times V)$ (up to a subsequence), it is easily seen that Theorem 6.1 holds true also for these eigenvectors.

Proof. In the course of the proof of Lemma 4.1 we obtained the following energy equality

$$
\begin{aligned}
\int_{\Omega} \int_{V} u_{\varepsilon} \psi_{\varepsilon}^{*} F_{\varepsilon}(q) \mathrm{d} x \mathrm{~d} v= & \frac{1}{2 \varepsilon} \int_{\Gamma_{+}}\left|u_{\varepsilon}\right|^{2} \psi_{\varepsilon} \psi_{\varepsilon}^{*} v \cdot n \mathrm{~d} v \mathrm{~d} \sigma+\frac{1}{2 \varepsilon^{2}} \int_{\Omega} \int_{V} \int_{V} \psi_{\varepsilon}^{*}(v) \sigma_{\infty}^{\varepsilon}\left(v^{\prime}, v\right) \psi_{\varepsilon}\left(v^{\prime}\right) \mid u_{\varepsilon}(v) \\
& -\left.u_{\varepsilon}\left(v^{\prime}\right)\right|^{2} \mathrm{~d} v \mathrm{~d} v^{\prime} \mathrm{d} x .
\end{aligned}
$$

Since $u_{\varepsilon}(x, v)$ converges strongly to $u^{0}(x)$ in $L^{2}(\Omega \times V)$ we have

$$
\lim _{\varepsilon \rightarrow 0} \int_{\Omega} \int_{V} u_{\varepsilon} \psi_{\varepsilon}^{*} F_{\varepsilon}(q) \mathrm{d} x \mathrm{~d} v=\int_{\Omega} u^{0} F(q) \mathrm{d} x=\int_{\Omega} D \nabla u^{0} \cdot \nabla u^{0} \mathrm{~d} x
$$

On the other hand, taking $\xi=\nabla u^{0}$ in Lemma 5.5 and using the definition of $u^{1}$ yields

$$
\int_{\Omega} D \nabla u^{0} \cdot \nabla u^{0} \mathrm{~d} x=\frac{1}{2} \int_{\Omega} \int_{Y} \int_{V} \int_{V^{\prime}} \sigma_{\infty} \psi^{*}(v) \psi(v)\left|u^{1}(v)-u^{1}\left(v^{\prime}\right)\right|^{2} \mathrm{~d} x \mathrm{~d} y \mathrm{~d} v \mathrm{~d} v^{\prime} .
$$

By the lower semi-continuity of two-scale convergence applied to $\varepsilon^{-1}\left(u_{\varepsilon}(v)-u_{\varepsilon}\left(v^{\prime}\right)\right)$, which two-scale converges to $\left(u^{1}(v)-u^{1}\left(v^{\prime}\right)\right)$, we obtain that

$$
\begin{array}{rl}
\liminf _{\varepsilon \rightarrow 0} \frac{1}{\varepsilon^{2}} \int_{\Omega} \int_{V} \int_{V^{\prime}} \sigma_{\infty}^{\varepsilon} \psi_{\varepsilon}^{*}(v) \psi_{\varepsilon}\left(v^{\prime}\right)\left|u_{\varepsilon}(v)-u_{\varepsilon}\left(v^{\prime}\right)\right|^{2} & \mathrm{~d} x \mathrm{~d} v \mathrm{~d} v^{\prime} \\
& \geq \frac{1}{2} \int_{\Omega} \int_{Y} \int_{V} \int_{V} \sigma_{\infty} \psi^{*} \psi\left|u^{1}(v)-u^{1}\left(v^{\prime}\right)\right|^{2} \mathrm{~d} x \mathrm{~d} y \mathrm{~d} v \mathrm{~d} v^{\prime} .
\end{array}
$$

Therefore, the energy balance implies that

$$
\lim _{\varepsilon \rightarrow 0} \frac{1}{\varepsilon} \int_{\Gamma_{+}}\left|u_{\varepsilon}\right|^{2} v \cdot n \psi \psi^{*} \mathrm{~d} \Gamma=0
$$

and

$$
\lim _{\varepsilon \rightarrow 0} \frac{1}{\varepsilon^{2}} \int_{\Omega} \int_{V} \int_{V^{\prime}} \sigma_{\infty}^{\varepsilon} \psi_{\varepsilon}^{*} \psi_{\varepsilon}\left|u_{\varepsilon}(v)-u_{\varepsilon}\left(v^{\prime}\right)\right|^{2}=\int_{\Omega} \int_{Y} \int_{V} \int_{V^{\prime}} \sigma_{\infty} \psi^{*} \psi\left|u^{1}(v)-u^{1}\left(v^{\prime}\right)\right|^{2} .
$$

Equation (43) is nothing but the strong two-scale convergence of $\varepsilon^{-1}\left(u_{\varepsilon}(v)-u_{\varepsilon}\left(v^{\prime}\right)\right)$ to $\left(u^{1}(v)-u^{1}\left(v^{\prime}\right)\right)$ (see Th. 1.8 in [2]). Since $u^{1}(v)$ is independent of $v^{\prime}$ and $u^{1}\left(v^{\prime}\right)$ of $v$, it implies the strong two-scale convergence of $\varepsilon^{-1}\left(u_{\varepsilon}-\int_{V} u_{\varepsilon}\right)$ to $u^{1}-\int_{V} u^{1}$. Since $u^{0}$ is smooth, $u^{1}(x, x / \varepsilon, v)$ is a well-defined function in $L^{2}(\Omega \times V)$, and as a result of Th. 1.8 in [2] we obtain the desired convergence.

A complete numerical analysis of the homogenization procedure in a realistic setting goes beyond the scope of this paper; we refer to [9] for such a presentation. Nevertheless we would like to discuss briefly some numerical examples that illustrate interesting features of the asymptotic behavior of the eigen-elements of (2). We consider here the two-dimensional approximation of an idealized nuclear reactor, which is a periodic square composed of $n \times n$ periodicity cells. Such cells, called assemblies, are themselves made of many fuel pins and control rods immersed in water. More precisely, each cell is a MOX assembly (MOX stands for a mixture of uranium and plutonium oxides) which is a very heterogeneous type of assembly (we shall not described them here, and we refer to [9] for more details). Two quantities have been of interest so far: the first eigenvalue $\lambda_{\varepsilon}^{1}$ and the corresponding positive eigenvector $\phi_{\varepsilon}$ of (2). Following the usual engineering notation, we define $k_{\text {eff }}=1 / \lambda_{\varepsilon}^{1}$. 
TABLE 1. Reference and reconstructed $k_{\text {eff }}$ for a MOX assembly.

\begin{tabular}{|c|c|c|c|}
\hline Number of assemblies & Reference $k_{\text {eff }}$ & Reconstructed $k_{\text {eff }}$ & error $\left(10^{-5}\right)$ \\
\hline $5 \times 5$ & 1.28495 & 1.28416 & 79 \\
\hline $10 \times 10$ & 1.29525 & 1.29513 & 12 \\
\hline $20 \times 20$ & 1.29791 & 1.29790 & 1 \\
\hline$\infty$ & 1.29883 & & \\
\hline
\end{tabular}

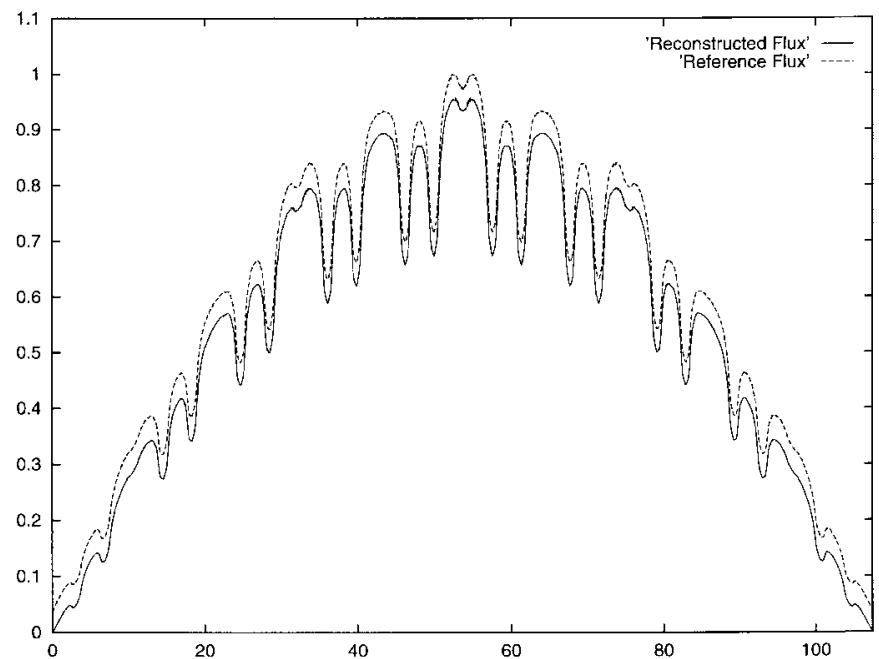

FiguRE 1. Reference flux and reconstructed flux in a core composed of $5 \times 5$ assemblies.

Table 1 gives the exact multiplication factor $k_{\text {eff }}$ for different values of the number $n$ of assemblies, and their associated diffusion approximation given by $\left(\lambda_{\infty}+\varepsilon^{2} \nu^{1}\right)^{-1}$. The error between these values, as seen in the last column of Table 1, has been given in the usual p.c.m. unit for nuclear reactors computations (1 p.c.m. is equal to $10^{-5}$ ). As a consequence of our analysis, the rate of convergence to the diffusion approximation of the exact eigenvalue is expected to be at least of order 2. A numerical estimate is given by

$$
v=\frac{\ln \frac{\mathrm{e}_{10}}{\mathrm{e}_{5}}}{\ln \frac{10}{5}} \simeq 2.71
$$

which is fairly well comparable to the theoretical value of 3 (obtained in [8] for symmetric cells), all the more since the number of assemblies is small in order not to heavily depend on the accuracy of the numerical computations (of the order of $10^{-5}$ for the eigenvalue).

In Figure 1 are plotted one-dimensional cross sections of the velocity-average of the exact first eigenvector $\int_{V} \phi_{\varepsilon}(x, v) \mathrm{d} v$ and of its diffusion approximation given by

$$
\left(\int_{V} \psi_{\infty}(x / \varepsilon, v) \mathrm{d} v\right) u^{1}(x)
$$

where $u^{1}$ is the first eigenvector of the homogenized diffusion equation (13). Let us emphasize that the shape of the reconstructed flux is extremely close to that of the exact transport solution (of course, since eigenvectors are defined up to a multiplicative constant, their normalization has been performed so that the difference between both flux is identical at the boundary of the cross section and at the point of maximal value). 


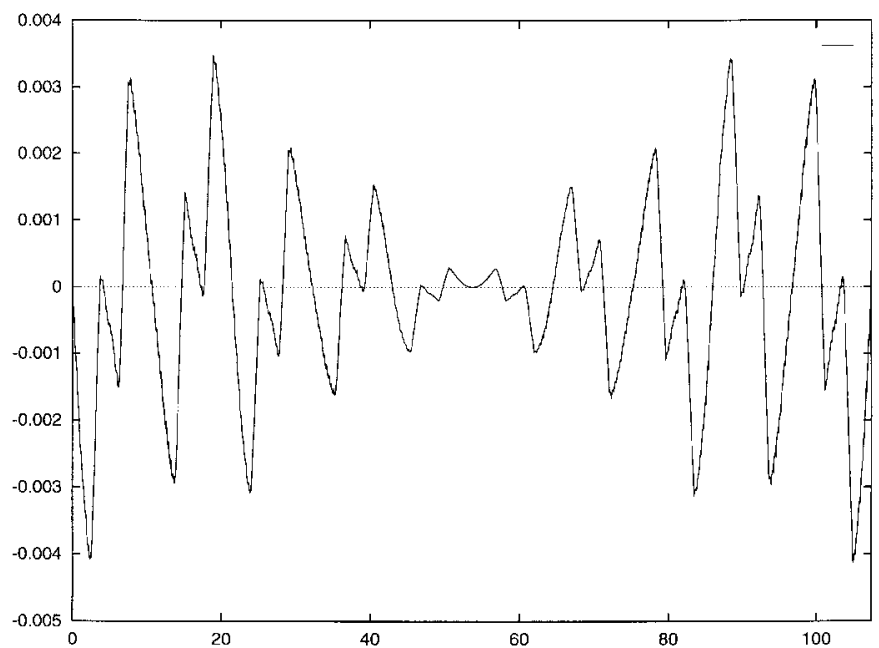

Figure 2. Periodic corrector for a flux normalized to 1 in a core composed of $5 \times 5$ assemblies.

However, whatever the choice of normalization, the error at the boundary of the domain remains of the order of $5 \%$ of the maximal value. This error can not be corrected by adding a periodic corrector term of order $\varepsilon$, but rather is due to a boundary layer effect. This is demonstrated by Figure 2 where the periodic corrector term, given by

$$
\varepsilon \sum_{j=1}^{N}\left(\int_{V} \theta^{j}(x / \varepsilon, v) \mathrm{d} v\right) \frac{\partial u^{1}}{\partial x_{j}}(x),
$$

is plotted. Since its contribution is of the order of 0.004 of the flux, whereas the error is of the order of 0.05 , this periodic corrector term is of no help, all the more since it vanishes at the boundary (using symmetry arguments, one can prove that at the boundary $\partial \Omega$ either $\partial u^{1} / \partial x_{j}$ vanishes owing to the Dirichlet boundary conditions, or $\theta^{j}$ is skew-symmetric in the sense that $\left.\int_{V} \theta^{j}(x / \varepsilon, v) \mathrm{d} v=0\right)$.

The error between the exact and reconstructed flux is of order $\varepsilon$. It is due to a boundary layer effect that we now explain. In the homogenization process, the absorbing boundary condition for the neutron flux is replaced by a boundary Dirichlet condition for the diffusion equation (vanishing flux on $\partial \Omega$ ). This amounts to saying that no neutrons either enter or exit the core asymptotically. However, for a fixed positive $\varepsilon$, this is not the case for the transport equation (2). Indeed, the absorbing boundary conditions in (2) implies that the incoming flux vanishes, while the outgoing flux is an unknown, and has no reason to cancel out. This phenomenon is the so-called leakage at the boundary of the core. Theorem 6.1 indicates that this leakage is of order smaller than $\varepsilon^{1 / 2}$, but a finer analysis shows that it is of order $\varepsilon[8]$. In practice, for most of the usual reactors (with about 150 assemblies), the neutron density at the boundary of the core (i.e. between the core and the reflector) is about $5 \%$ to $10 \%$ of the maximal density. Therefore quantitative numerical simulations have to account for this effect.

The highly heterogeneous structure of the transport density with respect to the velocity $v$ at the boundary of the core (no neutrons enter, whereas some exit) makes it very difficult to approximate by diffusion (in essence independent of the velocity). Surprisingly enough, this difficulty can be overcame in a very satisfactory way. In order to study this leakage, a boundary layer analysis is performed which allows to understand the anisotropic behavior of the neutron density at the boundary of the core. Such techniques have already been applied in the case of homogeneous media (or slowly varying media) [11,29]. In our situation, the neutron mean free path is of the same order than the medium heterogeneities. Therefore, the reactor can not be assumed to be locally homogeneous and a genuine multidimensional boundary layer analysis has to be done (see [8] in the simplified 
setting of the even parity transport formulation). This boundary layer can be replaced by a modification of the Dirichlet boundary condition which becomes a Robin boundary condition for the homogenized diffusion limit (featuring a so-called extrapolation length of order $\varepsilon$, characteristic of the boundary layer). Introducing this refinement in the homogenized model gives extremely satisfactory numerical results for the reconstructed flux, as well as for the leading eigenvalue (for details, see [9]).

The authors wish to thank B. Perthame for helpful discussions concerning the regularity theory of transport equations and X. Warin for his contribution to the numerical analysis presented here.

\section{REFERENCES}

[1] R. Alexandre and K. Hamdache, Homogénéisation d'équations cinétiques en milieu perforé. C.R. Acad. Sci. Paris Sér. I 313 (1991) 339-344.

[2] G. Allaire, Homogenization and two scale convergence. SIAM J. Math. Anal. 23 (1992) 1482-1518.

[3] G. Allaire and G. Bal, Homogénéisation d'une équation spectrale de transport neutronique. C.R. Acad. Sci. Paris Sér. I 325 (1997) 1043-1048.

[4] G. Allaire and Y. Capdeboscq, Homogenization of a spectral problem for a multigroup neutronic diffusion model. Comput. Methods Appl. Mech. Engrg. (to appear).

[5] G. Allaire and F. Malige, Analyse asymptotique spectrale d'un problème de diffusion neutronique. C.R. Acad. Sci. Paris Sér. I 324 (1997) 939-944.

[6] P. Anselone, Collectively compact operator approximation theory and applications to integral equations. Prentice-Hall, Englewood Cliffs, N.J. (1971).

[7] G. Bal, Couplage d'équations et homogénéisation en transport neutronique. Ph.D. thesis, University of Paris 6, France (1997).

[8] G. Bal, First-order Corrector for the Homogenization of the Criticality Eigenvalue Problem in the Even Parity Formulation of the Neutron Transport. SIAM J. Appl. Math. (to appear).

[9] G. Bal and X. Warin, An asymptotic analysis for the homogenization of P.W.R. assemblies - The transport case -. Nuclear Sci. Engng. (submitted).

[10] P. Benoist, Théorie du coefficient de diffusion des neutrons dans un réseau comportant des cavités. Note CEA-R 2278 (1964).

[11] A. Bensoussan, J.L. Lions and G. Papanicolaou, Boundary layers and homogenization of transport processes. Publ. RIMS Kyoto Univ. (1979) 53-157.

[12] J. Bussac and P. Reuss, Traité de neutronique. Hermann, Paris (1978).

[13] F. Chatelin, Spectral approximation of linear operators. Academic Press (1983).

[14] R. Dautray and J.L. Lions, Mathematical analysis and numerical methods for Science and Technology, Vol. 6. Springer Verlag, Berlin (1993).

[15] V. Deniz, The theory of neutron leakage in reactor lattices, in Handbook of nuclear reactor calculations, Vol II, Y. Ronen Ed. (1968) 409-508.

[16] L. Dumas and F. Golse, Homogenization of transport equations. Preprint No. R98003, University of Paris 6, LAN.

[17] E. Frenod and K. Hamdache, Homogenization of transport kinetic equation with oscillating potentials. Proc. Roy. Soc. Edinburgh Sect. A 126 (1996) 1247-1275.

[18] F. Golse, P.L. Lions, B. Perthame and R. Sentis, Regularity of the moments of the solution of a transport equation. J. Funct. Anal. 76 (1988) 110-125.

[19] T. Goudon and F. Poupaud (personal communication).

[20] E. Larsen, Neutron transport and diffusion in inhomogeneous media I. J. Math. Phys. 16 (1975) 1421-1427.

[21] E. Larsen, Neutron transport and diffusion in inhomogeneous media II. Nuclear Sci. Engng. 60 (1976) 357-368.

[22] E. Larsen and J. Keller, Asymptotic solution of neutron transport problems for small mean free paths. J. Math. Phys. 15 (1974) $75-81$.

[23] E. Larsen and M. Williams, Neutron Drift in Heterogeneous Media. Nuclear Sci. Engng. 65 (1978) $290-302$.

[24] F. Malige, Étude mathématique et numérique de l'homogénéisation des assemblages combustibles d'un cœur de réacteur nucléaire. Ph.D. thesis, École Polytechnique, France (1996).

[25] M. Mokhtar-Kharroubi, Les équations de la neutronique. Ph.D. thesis, University of Paris XIII, France (1987).

[26] G. Nguetseng, A general convergence result for a functional related to the theory of homogenization. SIAM J. Math. Anal. 20 (1989) 608-623.

[27] J. Planchard, Méthodes mathématiques en neutronique. Collection de la Direction des Études et Recherches d'EDF, Eyrolles (1995).

[28] F. Santosa and M. Vogelius, First-order corrections to the homogenized eigenvalues of a periodic composite medium. SIAM J. Appl. Math. 53 (1993) 1636-1668.

[29] R. Sentis, Study of the corrector of the eigenvalue of a transport operator. SIAM J. Math. Anal. 16 (1985) 151-166. 